\title{
The dynamics of Jupiter and Saturn in the gaseous proto-planetary disk
}

\author{
Alessandro Morbidelli \\ Aurélien Crida \\ Observatoire de la Côte d'Azur
}

Corresponding author:

Alessandro Morbidelli

$\mathrm{OCA}$

B.P. 4229

06304 Nice Cedex 4, France

email: morby@obs-nice.fr

Received __; accepted _ 


\begin{abstract}
We study the possibility that the mutual interactions between Jupiter and Saturn prevented Type II migration from driving these planets much closer to the Sun. Our work extends previous results by Masset and Snellgrove (2001), by exploring a wider set of initial conditions and disk parameters, and by using a new hydrodynamical code that properly describes for the global viscous evolution of the disk. Initially both planets migrate towards the Sun, and Saturn's migration tends to be faster. As a consequence, they eventually end up locked in a mean motion resonance. If this happens in the $2: 3$ resonance, the resonant motion is particularly stable, and the gaps opened by the planets in the disk may overlap. This causes a drastic change in the torque balance for the two planets, which substantially slows down the planets' inward migration. If the gap overlap is substantial, planet migration may even be stopped or reversed. As the widths of the gaps depend on disk viscosity and scale height, this mechanism is particularly efficient in low viscosity, cool disks. The initial locking of the planets in the 2:3 resonance is a likely outcome if Saturn formed at the edge of Jupiter's gap, but also if Saturn initially migrated rapidly from further away. We also explore the possibility of trapping in other resonances, and the subsequent evolutions. We discuss the compatibility of our results with the initial conditions adopted in Tsiganis et al. (2005) and Gomes et al. (2005) to explain the current orbital architecture of the giant planets and the origin of the Late Heavy Bombardment of the Moon.
\end{abstract}




\section{Introduction}

The general theory of planet-gas disk interactions (see for instance Lin and Papaloizou, 1979; Goldreich and Tremaine, 1980; Papaloizou and Lin, 1984; Lin and Papaloizou, 1986; Ward, 1986, 1997) predicts a systematic migration of the planets towards the central star. This prediction received a spectacular confirmation with the discovery of the first extra-solar planets, on orbits with semi-major axes comparable to or smaller than those of the terrestrial planets in our Solar System (the so-called hot and warm Jupiters).

Despite planet migration is undoubtedly a fact, it is not a general rule, or -at leastnot a rule without exceptions. In our Solar System, Jupiter and Saturn should not have migrated substantially, despite they evidently formed in a massive gaseous disk in order to accrete their hydrogen rich atmospheres. In fact, the existence of Uranus and Neptune outside of Saturn's orbit, and of the Kuiper belt beyond Neptune, constrains the inward migration of Jupiter and Saturn within a few AUs at most (probably much less; see below). In extra-solar systems, with the extension of the timescale of observations, new planets have been found at distances from the parent star comparable to that of Jupiter. Thus, it is important to study non-generic mechanisms that, in some cases, might stop planet migration, or slow it down significantly.

A new tight constrain on the orbits of Jupiter and Saturn at the time of disappearance of the gas disk comes from a recent model developed to explain the origin of the Late Heavy Bombardment (LHB) of the terrestrial planets (Gomes et al., 2005). In addition to the LHB, this model explains the current orbital architecture of the giant planets (orbital separations, eccentricities and mutual inclinations; Tsiganis et al., 2005) the origin and orbital distribution of the Trojans of Jupiter (Morbidelli et al., 2005) and the structure of

the Kuiper belt (Levison et al., 2007). If true, this model implies that, at the disappearance of the gas, the giant planets and the primordial trans-Neptunian planetesimal disk were 
originally in a compact configuration. This limits severely the radial migration of the planets during the preceeding phase, dominated by the interactions with the gas. Moreover, the orbits of the planets were quasi-circular, and the ratio of the orbital periods of Saturn and Jupiter was smaller than 2. Therefore, it is important to investigate whether these constraints are consistent with the dynamics of the planets in the gas disk, as far as we understand it.

A pioneer work in this direction has been done by Masset and Snellgrove (2001; MS01 hereafter). In that letter the authors presented the first numerical simulation of the evolution of Jupiter and Saturn in a gas disk. Saturn initially started at twice the heliocentric distance of Jupiter. After a phase of inward runaway migration, Saturn was captured into the 2:3 mean motion resonance with Jupiter. At that point, the planets reversed their migration, moving outward in parallel, while preserving their resonant relationship. This result is interesting for our purposes in two respects. First, it shows that a two-planet system in some configurations can avoid migration toward the central star. Second, the stable relative configuration achieved by Saturn and Jupiter is characterized by a ratio of orbital periods smaller than 2, as required by the LHB model discussed above.

Therefore, we think that it is timely to investigate more in detail the mechanism unveiled by MS01, in particular exploring a wider range of parameters. In fact, there are a few open issues on the validity of the mechanism and its consistency with the solar system structure that we need to address:

i) it is unclear how the mechanism depends on the adopted initial conditions. If the initial, inward runaway migration of Saturn is a crucial aspect, then the overall result might depend on the numerical resolution of the disk and on its initial state (namely, whether the planets are dropped into a virgin disk, or they are allowed to sculpt the disk for some time before they are let free to migrate). In fact, some researchers 
could not successfully reproduce the MS01 simulation (Kley, private communication), possibly because of these issues. Moreover, a wide range inward migration of Saturn might be inconsistent with the compact orbital architecture of the giant planets invoked by the LHB model.

ii) Jupiter -and to some lesser extent Saturn-, being a giant planet, undergoes type II migration. It is well known that this kind of migration follows the global evolution of the disk. The numerical algorithm used in MS01 could not model the evolution of the disk correctly, as it considered only an annulus of it, with wise -but nevertheless arbitrarily chosen- boundary conditions. In Crida et al. (2007) we have presented a new hybrid scheme that allows the computation of the global evolution of the disk, using a system of nested 1D and 2D grids. Consequently, this algorithm allows a correct simulation of Type II migration. It is true that MS01 argues that, once the planets are in the 2:3 resonance, they are no longer locked in the evolution of the disk (the reason for which they can move outward, despite the disk has a global motion towards the Sun). Nevertheless, the torque unbalance that MS01 measured depends critically on the mass of the disk inside the orbit of Jupiter, and the latter depends on the global evolution of the disk. In particular, it is known that giant planets can open cavities in the inner part of the disk (Rice et al., 2003; Varniere et al., 2006; Crida and Morbidelli, 2007). If this happened in this case, Jupiter and Saturn could not migrate outward. Thus, we think that it is important to re-simulate the dynamics of Jupiter and Saturn using our new, trustable algorithm.

iii) the evolution of Jupiter presented in MS01 is probably inconsistent with the solar system architecture. In fact, after Jupiter and Saturn lock in their mutual 2:3 resonance, their outward migration is rather fast. Jupiter increases its orbital radius by $\sim 40 \%$ in 1,000 orbits. If this really occurred in the Solar System, Jupiter would 
have been at some time in the middle of the asteroid belt. The properties of the asteroid belt (in particular the quite tight zoning of the taxonomic types) exclude this possibility. Thus, we need to find orbital evolutions that are much more stationary than the one presented in MS01.

iv) MS01 claim that the trapping of Jupiter and Saturn into the 2:3 resonance is the most likely outcome of their evolution in the gas disk. This might be problematic in the context of the LHB model (Gomes et al., 2005). That model argues that the ratio of Saturn's and Jupiter's orbital periods was smaller than 2, but requires that it was not too much smaller than this value. Otherwise, the mass of the planetesimal disk remaining after the disappearance of the gas would not have been sufficient to drive Saturn across the 1:2 resonance with Jupiter, which is required to trigger the planetary instability responsible for the origin of the LHB. Thus, it is important to investigate if other resonances between Jupiter and Saturn are possible in the context of the mechanism of MS01, or if there are possibilities to leave the resonance towards the end of the gas disk lifetime.

With these goals in mind, this paper is structured as follows. In Sect. 2 we briefly review MS01 result using a very similar simulation (and the same numerical code), and then we discuss the dependence of the results on the initial location of Saturn. In Sect. 3 we use the algorithm of Crida et al. (2007) to investigate how the dynamics of the Jupiter-Saturn couple depends on the disk's aspect ratio and viscosity, and also on numerical parameters such as disk's resolution and smoothing length. In Sect. 4 we briefly address the effect of the accretion of mass onto the planets, already partially discussed in MS01. In Sect. 5 we explore the dynamics for different values of the planet masses, to understand how generic the MS01 mechanism can be for extra-solar planet cases. In Sect. 6 we discuss possible ways to reconcile the MS01 mechanism with the LHB model of Gomes et al. Our conclusions are 
then recollected in the summary section.

\section{The Masset-Snellgrove mechanism}

Fig. 1 is a quite close reproduction of Fig. 1 in MS01. It has been obtained using the code presented in Masset (2000a,b) (the same as used in MS01), with similar parameters. Specifically, Jupiter is initially at $r=1$, while Saturn is at $r=2$. The disk extends from $r=0.3$ to 5 . It is modeled in two dimensions, using a grid with resolution 282 in radius and 325 in azimuth. Its initial surface density is uniform in radius and is equal to $6 \times 10^{-4}$ in our units (the mass of the Sun is 1 ), which corresponds to the surface density of the minimal mass nebula (Hayashi, 1981) if the unit of length corresponds to 5 AU. The boundary conditions allow outflow, but not inflow. We adopt an $\alpha$ prescription for the viscosity (Shakura and Sunyaev, 1973), with $\alpha=6 \times 10^{-3}$, and assume a constant aspect ratio $H / r=4 \%$ in the disk's equation of state.

Both Jupiter and Saturn initially migrate inward, as expected. The migration of Saturn accelerates exponentially, in a runaway -also called Type III- regime (Masset and Papaloizou, 2003) that is well fitted by an exponential curve up to $t \sim 100$. After a time

of about 100 Jovian initial orbital periods, Saturn crosses the 1:2 mean motion resonance (MMR) with Jupiter, because its migration is faster than the threshold below which the capture into resonance is certain in the 'adiabatic' approximation (Malhotra, 1993): $\left|\dot{a}_{S}\right| /\left(a_{S} \Omega_{S}\right)<<0.5 j(j+1) \mu_{J} e_{S}$, for the $j: j+1$ resonance, where $\mu_{J}$ is the mass ratio of Jupiter to the central object, and $a_{S}, e_{S}$ and $\Omega_{S}$ are Saturn's semi-major axis, eccentricity and angular velocity, respectively (MS01). Once within the 1:2 resonance, Saturn's runaway migration breaks. This happens because Saturn approaches the outer edge of Jupiter's gap; thus the disk inside its orbit starts to be partially depleted and consequently Saturn's coorbital mass deficit becomes smaller that the planet's own mass (Masset and Papaloizou, 
2003).

After this change in migration regime, Saturn's inward motion continues at a slower, approximately constant rate. After 280 initial orbital period, the migration of Jupiter stops and then it reverses. As they move in opposite directions, Jupiter and Saturn are eventually captured in their mutual 2:3 MMR, at $t=350$. After this event, the two planets migrate outward in parallel. The eccentricity of Saturn stabilizes around 0.012 and that of Jupiter around 0.003. It is worth noticing that the ratio of semi major axes of the two planets does not correspond to a 2:3 ratio of the Keplerian orbital periods. It rather corresponds to a $5: 8$ ratio. However, by looking at the behavior of the resonant angles, we have checked that the planets are really captured in the 2:3 MMR. The gravity of the disk displaces the mean motion resonances relative to the unperturbed keplerian location.

As we said in the introduction, it is an unsolved issue whether the initial evolution of Saturn, with its runaway migration and fast passage across the 1:2 MMR, plays a role in the subsequent dynamics. For instance, MS01 argue that the capture in the 2:3 MMR is favored by Saturn's eccentricity being enhanced during the previous 1:2 MMR crossing. Our understanding of planet formation is too limited to assess with confidence where Saturn formed. However, there is an emerging view that Saturn might have accreted its atmosphere (and therefore acquired the bulk of its mass) when it was already quite close to Jupiter. In fact, the immediate neighborhood of Jupiter's gap, being a local maximum of the disk's surface density, acts as an accumulation point of dust and small planetesimals (Haghighipour and Boss, 2003), and thus appears as a sweet spot for the growth of Saturn's core. Moreover, Saturn's core, independently of its formation location, might have suffered Type I migration until it was halted at the edge of Jupiter's gap, which acts as a planet trap (Masset et al., 2006), or in a mean motion resonance with Jupiter (Thommes, 2005). Thus, we believe that it is important to verify whether the MS01 mechanism can still work 
if Saturn is released in the proximity of Jupiter.

Fig. 2 shows the result of a simulation that differs from the previous one only for the initial location of Saturn (now at $r=1.4$ ). As one sees, after a short range migration, the planet is trapped into the 2:3 MMR, and then the evolution is the same as in the previous simulation. Thus, the migration reversal found in MS01 does not depend on the history of the previous migration. For this reason, and because of the arguments described above in favor of a close formation of Saturn, in the following simulations we will always release Saturn at a distance of 1.4 (which corresponds to the typical position of the edge of Jupiter's gap).

\section{Dynamics of Jupiter and Saturn as a function of the disk properties and simulation parameters}

To explore how the dynamics of Jupiter and Saturn is affected by the main parameters of the problem, we use, from now on, the numerical scheme described in Crida et al. (2007). In this scheme, the disk is represented using a system of $2 \mathrm{D}$ and $1 \mathrm{D}$ grids. The main portion of the disk, in which the planets evolve, is represented with a $2 \mathrm{D}$ grid in polar coordinates, as usual. The origin of the coordinates is the barycentre of the system. The inner part of the disk (ranging from the inner physical radius, e.g. the $\mathrm{X}$-wind truncation radius at a few tenths of $\mathrm{AU}$, to the inner boundary of the $2 \mathrm{D}$ grid) and the outer part of the disk (ranging from the outer boundary of the 2D grid to the physical outer edge, e.g. the photo-dissociation radius at hundreds of $\mathrm{AU}$ ) are represented with a $1 \mathrm{D}$ grid. The 1D grids have open outflow boundaries at the inner and outer physical edges, and exchange information with the $2 \mathrm{D}$ grid for the definition of realistic, time-dependent boundary conditions of the latter. The algorithm for the interfacing between the $1 \mathrm{D}$ and $2 \mathrm{D}$ grids is driven by the requirement that the angular momentum of the global system (the disk in the 
$2 \mathrm{D}$ section, plus the disk in the $1 \mathrm{D}$ section plus the planet-star system) is conserved. With this approach, the global viscous evolution of the disk and the local planet-disk interactions are both well described and the feedback of one on the other can be properly taken into account. Because the migration of giant planets depends on the global evolution of the disk, this code provides more realistic results than the usual algorithms, in which the evolution of the considered portion of the disk depends crucially on the adopted (arbitrary) boundary conditions. For more information and accuracy tests we refer the reader to Crida et al. (2007).

In all simulations presented below, the $2 \mathrm{D}$ grid is as before (from 0.3 to 5 , with $282 \times 325$ resolution). The inner $1 \mathrm{D}$ grid starts at $r=0.016$ and the outer $1 \mathrm{D}$ grid ends at $r=40$ (which corresponds to about $200 \mathrm{AU}$ in our units). They have the same radial resolution as the $2 \mathrm{D}$ grid. The initial surface density profile of the disk is of type $\Sigma(r)=3 \times 10^{-4} \exp \left(-r^{2} / 53\right)$, as illustrated with a dash-dotted line in Fig. 4, and is derived from the analysis of Guillot and Hueso (2006) for a disk evolving under the collapse of new matter onto the plane from the proto-stellar cloud, viscous evolution and photo-evaporation. The viscosity is assumed constant, for simplicity (we have verified, as MS01, that an $\alpha$ prescription for the viscosity would not change the results significantly, as the planets are very close to each other, although it can affect the global evolution of the disk).

Conversely to what we did in the previous section, we first let the planets evolve in the disk for 8,000 Jupiter orbits without feeling the disk's perturbations, assuming an aspect ratio $H / r=3 \%$ and a viscosity $\nu=10^{-5.5}$ (in our units, see above). This viscosity at $r=1$ corresponds to $\alpha=3.5 \times 10^{-3}$ in a Shakura and Sunyaev (1973) prescription. This simulation allows the planets to sculpt the disk, opening gaps around their orbits, and it sets a new surface density profile of the disk. When we do simulations with different disk parameters, we start from this profile, and let the planets evolve for additional 400 
Jovian orbits still without feeling the disk's perturbations, so that the disk profile adapts to the new situation. Only at this point we release the planets, letting them evolve under the effects of the disk and of their mutual perturbations. This procedure allows us to avoid possible spurious initial migrations, that might occur if the initial gas distribution is inconsistent with the presence of the planets.

\subsection{Dependence on the disk aspect ratio}

In a first series of runs, we have fixed the value of the viscosity $\left(\nu=10^{-5.5}\right.$, in our units), and we have studied the evolution of Jupiter and Saturn as a function of the disk aspect ratio $H / r$. MS01 found that the aspect ratio simply changes the outward migration speed, by a quantity proportional to $(H / r)^{-3}$. We find that the value of the aspect ratio can have a much more important impact on the dynamical evolution.

As Fig. 3 shows, if the aspect ratio is small $(3-4 \%)$, the evolution is similar to that previously considered. When released, Jupiter starts to migrate outward, while Saturn moves inward. After locking in the mutual 2:3 mean motion resonance, both planets move outward. In these cases, the migration is indeed faster if the disk is thinner, as found in MS01. However, for thicker disks, the evolution changes qualitatively. If the aspect ratio is $5 \%$, we find a quasi-stationary solution. After locking in the 2:3 MMR, both Jupiter and Saturn essentially do not migrate any more. Actually, Jupiter moves outward by only $1.5 \%$ in 2000 orbits. To our knowledge, this is the first quasi-stationary solution ever found for a system of giant planets in a fully evolving disk. If the disk thickness is increased to $6 \%$, both planets migrate inward, even after being captured into the 2:3 MMR. This migration is very slow, compared to that of an isolated Jupiter in the same disk. This sequence of behaviours relative to aspect ratio also suggests that, in a flaring disk, the planets might migrate until they find a position in the disk with the 'good' local aspect ratio that allows 
them not to migrate any more.

The reason of this parametric dependence of the evolution on $H / r$ is quite clear if one looks at the gas density profile at the moment when the planets are released (Fig. 4). As explained in Crida et al. (2006), the disk aspect ratio governs the width and the depth of the gaps opened by the planets. Therefore, if $H / r$ is large, there is more gas at the location of Saturn (i.e. just outside Jupiter's orbit) than in the case where $H / r$ is small. Consistently, there is slightly less gas inside of Jupiter's orbit (for $r<0.7$ ), because less material has been removed from the common gap formed by the two planets. As MS01 correctly pointed out, the direction of migration of Jupiter depends on the balance of the torques that the planet receives respectively from the disk inside its orbit (which pushes the planet outward) and and from the disk outside its orbit (which pushes the planet inward). In the case of an isolated planet, the torque from the outer disk is typically stronger, so that the planet migrates toward the Sun. But in this case, because the presence of Saturn depletes partially the outer disk, this torque is weakened. Obviously, it is weakened more if the gap at Saturn's position is deeper, namely if the disk aspect ratio is smaller, as visible in Fig. 4. Thus, if the aspect ratio is small enough, the torque received by Jupiter from the inner disk dominates that from the outer disk, and the planet migrates outward, feeling a net positive torque. Indeed, this is what we see happening in Fig. 3 .

The direction of migration of Jupiter determines the subsequent evolution of both planets, once they are locked in resonance. The planets have to move in parallel to preserve the resonant configuration. Therefore there is a competition between the net positive torque received by Jupiter and the net negative torque received by Saturn from the disk. Because these torques are monotonic functions of the planets' masses, and Jupiter is 3 times heavier than Saturn, in general the positive torque received by Jupiter dominates and the two planets move outward. In the case with $H / r=5 \%$, however, the net torque felt by Jupiter 
is close to zero, due to the specific density profile of the disk, and can be effectively canceled out by Saturn's torque. Thus, a non-migrating evolution is achieved after the planets lock in resonance.

For completeness and sake of clarity, in the remaining part of this sub-section we elaborate on some considerations already reported in sect. 2.4 of MS01. The principle of Type II migration is that, once a planet opens a gap, it positions itself inside the gap in order to balance the torques received from the inner and the outer parts of the disk. Then, locked into this equilibrium position, it is forced to follow the slow, global viscous evolution of the disk (Lin and Papaloizou, 1986), the latter described by the equations in Lynden-Bell and Pringle (1974). One could expect that the Jupiter-Saturn system should evolve in the same way. The outer migration of the pair of planets should approach Saturn to the outer edge of its gap, until Saturn feels a stronger torque that counterbalances the one received by Jupiter. In this situation the outward migration should stop, and the two planets should start to evolve towards the Sun, together with the disk. This, apparently, does not happen. For the disk parameters that we explore in this work, Saturn is not massive enough to open a clean gap (see Fig. 4). Thus, the conditions for a proper Type II migration are never fulfilled (see Crida and Morbidelli, 2007, for a discussion on Type II migration). If Saturn's radial migration is not the same as the natural radial motion of the gas, new material flows into its gap. However, the gaps of Jupiter and Saturn overlap, so that material flowing from the outer disk into the coorbital region of Saturn, after experiencing half of a horse-shoe trajectory relative to Saturn, can also perform half of a horse-shoe trajectory relative to Jupiter. The net result is a flow of matter from the outer part of the disk, through the Jupiter-Saturn common gap, into the inner part of the disk. To illustrate this process, Fig. 6 shows the surface density profile of the disk in the simulation with $H / r=3 \%$, at various times. Notice how, in first approximation, the Jupiter-Saturn gap simply 'shifts' through the disk. As the planets move outward, the disk is rebuilt inside the orbit of Jupiter and 
the surface density at the bottom of the gap increases as well. Both features are diagnostic of a mass flow through the planets system. In fact, in the code of Crida et al. (2007) that we use, the boundary conditions cannot act as a source of mass. Thus, an increase of the surface density in the inner part of the disk is possible only if there is an influx of mass from the outer disk.

The flow of gas has several effects. First, it unlocks the planets from the disk, allowing them to move against the gas stream. Second, it has positive feedbacks on the outer migration of the planets by (i) exerting a corotation torque on them, as it passes through their horseshoe regions and (ii) refurbishing the inner part of the disk, which exerts the positive torque on Jupiter discussed above. The signature of this feedback is well visible in the simulation with $H / r=3 \%$ in Fig. 3; the outward migration rate accelerates exponentially, which implies that there is a positive net torque that increases with the migration speed.

At this point, one might wonder whether the motion of the planets is dominated by the torque felt by Jupiter from the inner disk, or by the corotation torque exerted by the gas flowing through the planets' orbits. The flow of gas through the orbits of Saturn and Jupiter is the same; the size of the horseshoe regions of the two planets (and hence the magnitude of the corotation torque felt by each planet) is proportional to $M_{p}^{\gamma}$ for some $\gamma<1$; thus, the effect on the migration rate $\dot{a}_{p}$ of the planet is proportional to $M_{p}^{(\gamma-1)}$, namely it is larger for a lighter planet. So, if the corotation torque dominated the evolution of the planets, Saturn would be extracted from the resonance and would migrate away from Jupiter. As long as this does not happen (as in Fig. 3), the corotation torque cannot be the dominant force driving the planets' migration.

For a further indication that the corotation torque is weaker than the torque felt by Jupiter from the inner disk (Lindblad torque), we have done another simulation, still with 
$H / r=3 \%$ and $\nu=10^{-5.5}$, but with an initial gas density reduced by a factor of 2 . The Lindblad torque scales with the gas density. Conversely, the corotation torque does not scale simply with the gas density because a component of it depends on the the radial speed of the planet relative to the gas, which in turn also depends on the gas density (Masset and Papaloizou, 2003). Thus, if the Lindblad torque dominates, we expect the planets to have the same evolution, just a factor of 2 slower. If the corotation torque dominates, the change in the dynamics can not be trivially reduced to a simple scaling on time. Fig. 5 shows the result. The black curves show the evolution of Jupiter and Saturn in the nominal gas disk. The grey curves show the evolution of the planets in a disk with half the initial density. In plotting this second pair of curves, the time-span measured relative to the release time (400 orbits) has been divided by two. The black and grey curves superpose almost perfectly. This suggests that the Lindblad torque is stronger than the corotation torque.

\subsection{Dependence on the simulation's technical parameters}

Before proceeding further with our exploration of the dynamical evolution of Jupiter and Saturn, we check the impact of some technical parameters used in the simulation: specifically the smoothing length for the gravitational potential and the grid resolution for the disk.

As usual in 2 dimensional hydro-dynamical simulations, the equations of motions are regularized in the vicinity of the planet by modifying the gravitational potential energy $U(\Delta)=-\left(M_{p} m\right) / \Delta$ into $U_{\rho}(\Delta)=-\left(M_{p} m\right) / \sqrt{\Delta^{2}+\rho^{2}}$, where $\Delta$ is the distance between the planet of mass $M_{p}$ and a fluid element of mass $m$, and $\rho$ is called the smoothing length. The choice of an appropriate value for the smoothing length is the subject of a vast debate. Essentially, two recipes are used: either $\rho$ is chosen proportionally to the Hill radius of the 
planet, or proportionally to the local thickness of the disk.

In the simulations presented above, our choice of $\rho$ was equal to $60 \%$ of the planet's Hill radius $R_{H}=a_{p}\left(M_{p} / 3\right)^{1 / 3}$, where $a_{p}$ is the semi major axis of the planet and $M_{p}$ is normalized relative to the mass of the star. We have decided to redo the simulation with $H / r=3 \%$, adopting $\rho=0.7 H$, where $H$ is the thickness of the disk at the distance of the planet (namely $0.03 a_{p}$ ). In this case, the new value of $\rho$ for Saturn and Jupiter is, respectively, $73 \%$ and $50 \%$ of those previously adopted. The new simulation is compared with the previous one in Fig. 7, Because the simulations do not start exactly in the same way, for a more meaningful comparison we have plotted the evolution of the planets only from the time $T_{0}$ at which Saturn starts its outward migration (this time is slightly different in the two simulations) and we have renormalized the semi major axes of the planets by the semi major axis of Jupiter at $T_{0}$. As one sees, the difference is not very big. Using the new value of $\rho$ leads to a slightly faster migration. The reason is that Saturn opens a wider and deeper gap in the new simulation, because the smaller value of the smoothing length is equivalent to an enhancement of its gravitational potential. As we have seen before, a deeper gap at Saturn's location increases the unbalance of the torques exerted on Jupiter from the inner and the outer parts of the disk, and hence leads to a faster outward migration speed. We have performed all the simulations of Fig. 3 with the new prescription of the smoothing length. None of the simulations changes significantly. In particular we still find a quasi-stationary, non-migrating evolution in the case with $H / r=5 \%$ (Jupiter now migrates outward only by $0.5 \%$ in 2,000 orbits), and an inward migration in the case of $H / r=6 \%$. Because the choice of $\rho$ based from the local thickness of the disk seems to us somewhat more physically motivated, we will adopt this prescription in all the simulations presented further in this paper.

Whatever the choice of $\rho$ above $\left(0.6 R_{H}\right.$ or $\left.0.7 H\right)$, the smoothing length is always a big 
fraction of the planet's Hill radius. Thus, it is interesting to explore what would happen if we chose a much shorter smoothing length. In Fig. 8 we compare the simulation with $H / r=4 \%$ and $\rho=0.6 R_{H}$ (black curves, already illustrated in Fig. 3i) with one with the same disk parameters, but $\rho=0.25 R_{H}$ (grey curves) and one with the same prescription of $\rho$ but where we have nullified the torques exerted on the planet(s) by the gas inside their respective Hill spheres (light grey curves). The exclusion of the torques from the regions neighboring the planets is never implemented in all other simulations presented in this paper. As one sees, the planets' migration rates depend quite strongly on the adopted prescription for smoothing and torque calculation. This is because, if $\rho$ is small, Saturn opens a deeper gap at its location, which enhances the unbalance of the torques felt by Jupiter. What is important, however, is that in all cases the migration is outward. This, once again, shows the robustness of the MS01 mechanism.

The resolution of the grid used to represent the disk can also have, in principle, an important impact on the evolution of the system. In particular it can affect the corotation torque that, as we have seen, plays a role in the outward migration of the planets. To test the effects of the grid resolution, we have repeated the simulation with $H / r=3 \%$ and $\rho=0.7 \mathrm{H}$, increasing by a factor of two both the radial and azimuthal resolutions of the $2 \mathrm{D}$ grid, and the radial resolution of the 1D grids. The new simulation is also plotted in Fig. 7 , As one sees, the difference with respect to the simulation with our nominal resolution is negligible. Given the computational cost of the high resolution simulation, we will continue to use $282 \times 325$ cells in the $2 \mathrm{D}$ grid in the subsequent experiments.

Another technical issue concerns the initial condition for the gas distribution. As we said above, we start from a gas profile carved by the planets on fixed orbits in a simulation spanning 8,000 periods at $r=1$, with $H / r=3 \%, \nu=10^{-5.5}$ and $\rho=0.6 R_{H}$. However, when we change the parameters of the simulation, we only wait for additional 400 orbital periods 
before letting the planets free to evolve. This second delay might not be long enough for the gas to respond to the new conditions, possibly introducing artefacts in the subsequent planet dynamics. To check if this is indeed the case, we plot in Fig. 9 two simulations, for $H / r=4 \%, \nu=10^{-5.5}$ and $\rho=0.7 H$. In one simulation (black curves) the planets have been released after 400 orbits, as usual; in the second simulation, the planets have been released after 5,000 orbits. The evolutions after the release time match so perfectly that, in order to see the two sets of curves we had to downshift the gray ones by $1 \%$ ! Thus, we conclude that our relatively short rlelease time of 400 orbits does not introduce significant artefacts.

\subsection{Dependence on the disk viscosity}

We have done a series of simulations, changing the value of the viscosity, from $\nu=10^{-6}$ to $2 \times 10^{-5}$ in our units. The disk aspect ratio is $4 \%$ in all simulations. Thus, at $r=1$,

these viscosities correspond to $\alpha$ ranging from $6.25 \times 10^{-4}$ to $1.25 \times 10^{-2}$, in a Shakura and Sunyaev (1973) prescription. As usual, Saturn starts at $r=1.4$ and Jupiter at $r=1$. The results are illustrated in Fig. 10.

For a viscosity $\nu=10^{-6}$, Jupiter migrates outward after it has been released. Saturn initially migrates inward and, after being locked in the 2:3 MMR with Jupiter, the two planets migrate outward in parallel. For a viscosity $\nu=5 \times 10^{-6}$, the evolution is qualitatively similar. The outward migration speed, however, is faster than in the previous case. The reason is that the inner edge of Jupiter's gap is further away from the planet in the $\nu=10^{-6}$ case than in the $\nu=5 \times 10^{-6}$ case (see Fig. 11), so that the positive torque felt from the inner disk is weaker in the first case.

For a viscosity $\nu=10^{-5}$, Saturn -when released- has some erratic motion, which is slightly outward, on average, until $T=1200$. During this time-span, Jupiter, which is also 
migrating outward, approaches Saturn. Eventually Saturn has a short inward migration and is captured in the 2:3 MMR with Jupiter, and the two planets migrate outward together. Their common outward migration is slower than in the previous cases. If the viscosity is increased to $2 \times 10^{-5}$, as soon as released Saturn migrates outward. Jupiter in the meantime migrates inward. The mutual 1:2 MMR is crossed at $T=880$. The eccentricity enhancement that results from this resonance crossing breaks Saturn's outward migration. The planet starts a 'normal' inward migration, at a rate comparable to that of Jupiter. The two planets are close to the 1:2 MMR, but not locked in it. The resonant angles are in fact in circulation. The reason for the initial behavior of Saturn in these two simulations is most likely due to the corotation torque. As Fig. 11] illustrates, for these values of the viscosity there is quite a large amount of gas at Saturn's location, and the outer edge of Saturn's gap is very close to the planet. More importantly, Jupiter's gap becomes shallower. This reveals that, even before that the planets are released, there is an important flow of gas from the outer part of the disk, through the planetary orbits, towards the inner part of the disk. This flow exerts a corotation torque on each planet, which, as we discussed above, has stronger effects on Saturn.

Putting together these results with those of sect. 3.1, we conclude that the mechanism of MS01 works for a large range of values of aspect ratio and viscosity of the disk. Whenever the disk is enough thin and of low viscosity, Jupiter and Saturn can have a common outward migration, once locked in the 2:3 MMR. Finding a quasi-stationary solution, however, is more delicate. If the disk is relatively thick ( $5 \%$ and, presumably, more), a quasi-stationary solution can be found for some value of the viscosity. Conversely, if the disk is thin (4\% or, presumably, less), a quasi-stationary solution may not be found. The reason is that, if the disk's aspect ratio is decreased, in principle the viscosity needs to be increased in order to maintain a density at Saturn's location that is sufficiently large to exert on Jupiter a torque that counter-balances the one received by the planet from the inner disk. This larger 
viscosity, however, tends to destabilize Saturn, because it generates a stronger flow that exerts an more important corotation torque on the planet.

\section{The effect of mass accretion onto the planets}

In all previous simulations, the mass of the planets was kept constant with time. Lubow et al. (1999) and Kley (1999) showed that the accretion of mass by Jovian or sub-Jovian planets is non negligible for most values of the disk's parameters. The investigation of the effects of mass accretion onto the planets is therefore interesting. Mass accretion exerts additional torques onto the planets and breaks the flow of the gas across the planetary orbits. So, in principle it could modify the dynamics significantly.

MS01 already explored the effect of mass accretion onto Jupiter, and found that it is negligible even from the quantitative point of view. Here we consider also the effect of mass accretion onto Saturn, which might have a larger impact on the dynamics. Our understanding on how planet accretion proceeds, and how it stops, is still too vague to be able to assert a priori which planet should have had a more important mass growth rate.

As MS01, we have implemented mass accretion onto the planets following the recipe of Kley (1999). It consists in removing a fraction of the material in the Hill sphere of the planet and adding it to the mass of the planet. The amount which is removed in one time-unit is imposed as an input parameter. More specifically, we apply the input removal rate in the inner Hill sphere (extended up to $45 \%$ of the Hill radius $R_{H}$ ); we apply $2 / 3$ of the removal rate in the region from 0.45 to $0.75 R_{H}$ and no removal rate in the region beyond $0.75 R_{H}$. We have done 6 simulations, with three removal rates applied to Saturn only or both Jupiter and Saturn. The removal rates, (expressed as fraction of mass removed in the unit of time, which is $1 / 2 \pi$ of the initial Jupiter's orbital period) are $0.1,1$ and 5 , as in Kley 
(1999). All the simulations started from an intermediate state achieved in the simulation with $H / r=3 \%, \nu=10^{-5.5}$ (equivalent to $\alpha=3.5 \times 10^{-3}$ ) and $\rho=0.7 H$ (already presented in Fig. (7), precisely after a time corresponding to 900 initial Jovian orbital periods after the release of the planets.

Figure 12 shows the result in the case of an accretion rate of 1 applied on Saturn only and compares it with the nominal simulation that we started from, where no accretion was allowed. We notice that the outward migration rate of Saturn and Jupiter (up to 1700 orbital periods) is significantly smaller. During this time, the eccentricity of Saturn is larger than in the case without accretion $(\sim 0.05$ instead of $\sim 0.02)$, which means that Saturn is offering a stronger resistance to the outward push exerted by Jupiter through the 2:3 MMR. This is most likely due to the fact that Saturn is growing in mass, so that the negative torque that it receives from the outer part of the disk increases. In fact, from $t=900$ to $t=1700$, Saturn doubles it mass, in an essentially linear mode.

At $t=1700$ the dynamical evolution changes abruptly. The mass growth of Saturn is accelerated, so that the planet reaches one Jupiter mass at $t=1800$. This abrupt flow of mass onto the planet, essentially from the outer disk, exerts a strong positive torque. Therefore Saturn is extracted from the 2:3 MMR with Jupiter and runs away from it. Once separated from Jupiter, 'Saturn' starts an inward, Type II-like migration, despite of the positive torque due to the accretion of gas, which is still ongoing.

The simulation where the mass of Jupiter is also allowed to grow, is essentially identical to the one presented in Fig. 12. We note in passing that during the linear growth regime, while the mass of Saturn doubles, the mass of Jupiter increases by only $15 \%$. This shows that neglecting the growth of Saturn while allowing the growth of Jupiter is not justified. We also remark that the growth of the planets does not stall until they reach a mass of several Jupiter masses. This stresses the unsolved problem of explaining the final masses of 
the giant planets of the solar system (and of extra-solar systems in general).

The simulations with a smaller (0.1) or larger (5) removal rate parameter behave essentially like that presented in Fig. 12, Obviously, during the linear mass growth regime, the deviation with respect to the nominal simulation without mass accretion is smaller in the first case and larger in the second case. Even in the case with a removal rate of 5, though, we observe an outer migration of Jupiter of Saturn. This implies that this kind of dynamical evolution is robust with respect to the accretion rate, unless the latter is very high.

\section{Generic two-planet dynamics: dependence on the individual masses and mass ratio}

Although this paper is devoted to the evolution of Jupiter and Saturn, it is interesting to do a quick exploration of how the dynamics changes with the masses of the planets. We have done three simulations, all with $H / r=5 \%, \nu=10^{-5.5}$ (corresponding to $\alpha=1.25 \times 10^{-3}$ ) and $\rho=0.7 H$ (these parameters corresponds to the quasi-stationary solution for the Jupiter-Saturn system): the first one assumes that the inner planet has the mass of Saturn and the outer one has the mass of Jupiter; the second one assumes both masses are equal to one Jupiter mass; the third simulation multiplies the masses of the real planets by a factor of three.

The first two simulations give no surprises. As we explained in sect. 3.1, the outward migration is possible only if the inner planet is more massive than the outer one. Otherwise the balance between the positive torque felt by the inner planet and the negative torque felt by the outer planet is in favor of the latter one. In fact, in both the first and the second simulation the planets migrate inward. Initially, the outer planet migrates faster than the 
inner one, so that the two planets get captured in the 2:3 MMR after some time.

The third simulation is the most interesting. In this case the mass ratio is the same as in the Jupiter-Saturn case, favoring an outward migration. However, because the outer planet is more massive than Saturn, it may be more difficult to unlock the evolution of the planetary system from the evolution of the gas, which favors an inward migration. So, the result of this experiment is not evident a priori. Fig. 13 shows the outcome. When the planets are released (as usual after 400 orbital periods of the inner planet), the inner planet starts to move outward as expected. The outer planet remains essentially on the spot. Before that a resonant configuration is achieved, the planets destabilize each other, because their separation corresponds to less than 3 mutual Hill radii (a mutual Hill radius is defined as $\left[\left(a_{1}+a_{2}\right) / 2\right]\left[\left(M_{1}+M_{2}\right) / 3\right]^{1 / 3}$, where $a_{1}, a_{2}$ are the semi major axes and $M_{1}, M_{2}$ the masses). As a result of this instability, at $t=600$ the outer planet is propelled outward on an orbit with eccentricity equal to 0.25 , and the inner planet is kicked inward, onto an orbit with eccentricity equal to 0.1. Because of the large masses of the planets, the two gaps still partially overlap. Therefore, the inner planet feels a net positive torque, and the outer planet a net inward torque and, at $t=700-800$, they start to migrate in converging directions. During this time, their orbital eccentricities are damped down to less than 0.05. At $t=1000$ the planets are captured in their mutual 1:2 MMR. For a while after the resonant capture, the two planets move outward, but then eventually they stop, in a sort of quasi-stationary configuration. Our interpretation is that the gap of the outer planet is much more impermeable to the gas flow than in Saturn's case. Consequently, under the push felt from the inner planet, the outer planet simply approaches the edge of its gap and modifies its profile until the torque that it receives from the outer disk can counterbalance the torque from the inner planet. This stops the migration.

In conclusion, the mechanism proposed in MS01 is not necessarily specific to our 
solar system. It can apply to extra-solar planetary systems but only at given, stringent conditions: (i) the outer planet has to be significantly less massive than the inner one and (ii) the planets have to be locked in a resonance characterized by an orbital separation that is sufficiently small to allow the overlapping of the respective gaps. All of the 20 multi-planets extra-solar systems discovered so far should have suffered a wide range migration, as suggested by the close proximity of the planets to the central star (typically, the inner planet is within 1.5-2 AU and the outer planet within 4 AU). So, we should expect that the mechanism of MS01 did not work in these systems. In fact, in 13 cases criterion (i) is not fulfilled. In the remaining cases the planets are too separated, with ratios of orbital periods larger than 3, so that it is unlikely that they have ever been locked in resonances with small orbital separation in the past. We predict that extra-solar systems satisfying both conditions (i) and (ii) will be discovered in the future, when the observation time-span will become long enough to allow the detection of distant planets that did not migrate significantly.

\section{Possible ratios of orbital periods of Jupiter and Saturn}

In all the simulations reported above, as well as in those of MS01, whenever Jupiter and Saturn are in a configuration that prevents their migration towards the Sun, they are locked in the 2:3 MMR. This supports the idea, proposed in Tsiganis et al. (2005) and Gomes et al. (2005), that - at the end of the gas disk phase- the system of the giant planets in our solar system was very compact (i.e. characterized by small separations between the planets' orbits). However, from the quantitative point of view, our results do not support directly the initial conditions adopted in Tsiganis et al. and Gomes et al. The initial ratio between the orbital periods of Saturn and Jupiter in that model was 1.8-1.9. The exact value is not important, but it is required that it is close to 2, so that Saturn can cross the 
1:2 MMR with Jupiter in $\sim 650$ My (the time of the Late Heavy Bombardment) due to its interaction with the remaining planetesimal disk. If, at the end of the gas disk phase, the ratio of orbital periods had been close to 1.5, it is unlikely that this would have happened (unless a very massive planetesimal disk is assumed, but this would lead to other problems concerning the evolution of Uranus and Neptune). Therefore, in this section we explore different ways to reconcile the MS01 mechanism with the initial conditions of the LHB model.

In principle, it is not necessary that Saturn and Jupiter are locked in the 2:3 MMR in order to prevent their inward migration. Other resonances, characterized by a larger ratio of orbital periods, may work, provided that the gaps formed in the disk by the two planets are wide enough to overlap. This would give a constraint on the maximal viscosity and scale height of the disk for each chosen resonant configuration. Reality, however, is not that simple, because the resonances located between the 2:3 and 1:2 MMR are much thinner than first order resonances and they may be characterized by unstable motion. So, the possibility of capture and permanence of the planets in these resonances is not guaranteed, a priori.

We have done a series of 5 simulations, starting Saturn at a distance of 1.5 (Jupiter being initially at 1 , as usual, so that the initial ratio of the orbital periods is 1.84), in disks with aspect ratio of $3.5 \%$ and viscosities in the range $1-3 \times 10^{-6}$. In all simulations we have observed only captures in the 3:5 MMR, which led to a quasi-stationary evolution or a slow outward migration of the giant planets. However, in all cases, once captured in the resonance, the eccentricity of Saturn grew above 0.1 in about 150 initial Jovian orbital periods. This led to an instability of the planetary motion, which eventually led to a phase of violent scattering among the planets. Thus, we conclude that resonances of order larger than 1, located in between the 2:3 and 1:2 MMR are not viable for a long phase of 
quiescent, non-migrating evolution. They either don't capture the planets, or lead to an unstable motion after a short timescale.

We have also done two simulations with Saturn initially at a distance of $\sim 1.65$ (initial ratio with Jupiter's orbital period of $\sim 2.1$ ), in a disk with $H / r=3 \%$ and viscosity of $5 \times 10^{-6}$. In both cases we have obtained capture in the 1:2 MMR, and a subsequent quasi-stationary evolution of the semi major axes of the two planets. This shows that the passage across the resonance without capture observed in MS01 (and in Fig. 1 above) was due to the fact that Saturn was migrating very fast. Our initial conditions and the low viscosity of the disk allow a slower migration and a more gradual growth of the eccentricity, which favor capture. Once captured in the resonance, despite the eccentricities of the planets are not negligible, the orbital evolution of the planets looks stable. We have not found any obvious way of extracting the planets from the resonance after some time, and delivering them on orbits with orbital period ratio smaller than 2. So, we doubt that a capture in the 1:2 MMR during the gas disk phase may be compatible with the initial conditions of the LHB model.

Finally, we have studied the possibility that Saturn is extracted from the 2:3 MMR with Jupiter, after a long phase of quasi-stationary evolution, and is transported to larger semi major axis, approaching the 1:2 MMR.

A first idea is that, as the surface density of the disk decreases during the disk dissipation phase, the planetary motion might become unstable so that the planets push each other onto more widely separated orbits. We have rapidly discarded this possibility, because a 'stability map' shows that the Jupiter-Saturn system at low eccentricity is stable if the ratio of the orbital periods is larger than 1.45 (Gayon, private communication).

A second idea is suggested by the simulation presented in Fig. 12, The simulation should be considered only at a qualitative level for several reasons: accretion was started 
when Saturn already had one Saturn's mass, so that the final mass of the planet is larger than the real one; the prescription used for mass accretion was ad-hoc and idealized. Nevertheless, the simulation shows that rapid accretion of mass onto a planet exerts a positive torque that can extract the planet from the resonance. For instance, in Fig. 12 the ratio of orbital periods of Saturn and Jupiter at $t-T_{0}=1800$ is 1.85 , consistent with the initial conditions of the LHB model. Of course, once the planets are extracted from the resonance, their orbital evolution is no longer at equilibrium, and migration is resumed. Thus, to advocate a final position of the planets close to the 1:2 MMR, one has to assume that the disk disappeared 'at the right time'. Our understanding of planetary growth is still too poor to draw definite conclusions. However, the moderate mass of Saturn may be an indication that its rapid growth was indeed aborted by the disappearance of the disk (Pollack et al., 1996). Notice however that, if the growth of Saturn is really as rapid as the simulation shows (0.4 Jupiter masses in 700 orbits), the nebula has to dissipate on a timescale of $\sim 10,000 \mathrm{y}$, otherwise Saturn would have become too massive.

A further, possibly more promising idea, concerns the evolution of the viscosity of the disk. As we have seen in sect. 3.3, the MS01 mechanism works only if the viscosity is sufficiently small. If the viscosity exceeds some value, Saturn can be extracted from the resonance in a runaway migration mode (see Fig. 10). Thus, it is interesting to explore the dynamics of Jupiter and Saturn in the case of a disk whose viscosity increases with time. In principle, there are a few reasons to believe that the disk's viscosity might grow towards the end of the disk's lifetime. If the origin of viscosity is MHD turbulence (Balbus and Hawley, 1991), the viscosity depends on the ionization of the disk. A sufficiently massive disk is optically thick, so that the radiation from the star cannot penetrate in the disk and the gas is not ionized. Thus, a dead zone can exist inside the disk, at a typical distance from a few to a few tens of AU, where MRI turbulence is not sustained and therefore the viscosity is very small (Gammie, 1996). The giant planets might very well have formed in such a dead 
zone. When the disk starts to disappear, the radiation of the star can penetrate deeper into the disk, ionizing the disk on the mid-plane at larger heliocentric distance. The dead zone is re-activated, which causes an important enhancement of the local viscosity. Moreover, dust tends to have chemical bonds with the ions, subtracting them from the gas. Thus, even if undergoing the ionizing effect of the stellar radiation, a disk might not exhibit MHD turbulence if a sufficient amount of dust is present (Ilgner and Nelson, 2006a,b). As time passes, most of the dust is accreted in planetesimals, and therefore cannot subtract ions as efficiently as before. Therefore, a late disk should be increasingly coupled to the magnetic field and be characterized by a more violent turbulence and stronger viscosity.

Motivated by these considerations, we have designed the following experiment. We considered the simulation with Jupiter initially at $r=1$, Saturn at $r=1.4$ and a disk with $H / r=5 \%$ and $\nu=10^{-5.5}$ (corresponding to $\alpha=1.25 \times 10^{-3}$ ), performed assuming a smoothing length $\rho=0.7 \mathrm{H}$. In this simulation, after capture in the mutual 2:3 MMR, Jupiter and Saturn exhibit a remarkable quasi-stationary solution (see Fig. 14 up to $t=3200)$. At $t=3200$ we started to increase the viscosity of the disk, at the rate of $10^{-9}$ per unit of time (we remind that in our units the orbital period at $r=1$ is $2 \pi$ ). This rate is totally arbitrary, and not justified by any astrophysical considerations. As a consequence of the increase in viscosity, the gaps formed by the planets become narrower and overlap more marginally. Thus, the shape of the gap formed by Jupiter becomes more symmetric with respect to the position of the planet, so that the torque received by Jupiter from the outer part of the disk starts to dominate over that from the inner part of the disk. Consequently Jupiter starts to migrate towards the Sun. The migration rate increases with increasing viscosity. As the resonance with Jupiter moves inward, Saturn also migrates towards the Sun, but at a smaller rate. In fact, the flow of gas from the outer disk towards Jupiter exerts a corotation torque on Saturn, slowing down its inward migration. This extracts Saturn from the 2:3 MMR. As the viscosity increases, the corotation torque becomes stronger, 
and eventually Saturn starts an outward runaway migration. At $t=7200$ Saturn is very close to the 1:2 MMR with Jupiter, as required in the initial conditions of the LHB model. The eccentricities of Jupiter and Saturn are very low, less than 0.005 and 0.01 respectively, which is also consistent with the LHB model. At that time, the viscosity of the disk is $\nu=3.1 \times 10^{-5}$. Given that the aspect ratio is $5 \%$, this viscosity would correspond to a value of $\alpha \sim 10^{-2}$, which is still reasonable. Obviously, to support the initial conditions of the LHB model, one has to assume that the disk disappears at that time. If this were not the case, and the viscosity kept growing, Saturn would cross the 1:2 MMR with Jupiter. Notice that, overall, Jupiter has an inward migration that covers only $20 \%$ of its initial heliocentric distance. Thus, in this scenario, to justify its current position, Jupiter should have formed at about 6.5 AU (and Saturn at about 8.5 AU, to end up, more or less, at the same position). These ranges of migration are moderate, and do not violate, a priori, any of the constraints imposed by the current solar system architecture.

Again, we think that this simulation should be considered only at a qualitative level. Our knowledge of the evolution of the disk close to its disappearance is too approximated to be able to build a realistic simulation. Fig. 14 is presented simply to show that it is possible, in principle, to release the planets on non-resonant orbits after that they have spent most of the disk lifetime on resonant, non-migrating ones. Obviously, making the bridge between the formation of the planets, their dynamics in the gas disk, and their subsequent evolution in the planetesimal disk remains an open, crucial problem that goes beyond the scopes of this work.

\section{Summary}

In this paper we have analyzed in detail, by performing many numerical simulations, the mechanism proposed by Masset and Snellgrove (2001) to explain why Jupiter and 
Saturn did not migrate towards the Sun. The simulations have been done with a new simulation scheme (Crida et al., 2007), that is particularly suitable to study the migration of the giant planets. We confirmed that, if Jupiter and Saturn are locked into their mutual 2:3 MMR and the disk's viscosity and aspect ratio are sufficiently small, the planets do not migrate toward the Sun. The mechanism is robust with respect to grid resolution used for the disk, the smoothing length used for the regularization of the gravitational potential, and the accretion of mass onto the planets. In most cases, the planets migrate outward, which is not a viable evolution in our solar system, because it would imply that Jupiter was in the asteroid belt in the past. However, there is a range of values of viscosity and disk's scale height such that, once in resonance, the planets have a quasi-stationary evolution during which their semi major axes remain practically constant. We argue that Jupiter and Saturn actually followed this kind of evolution.

In general terms for a pair of planets, a quasi-stationary solution can be found only if the outer planet is significantly less massive than the inner one, and if the planets are locked in a resonance characterized by a small orbital separation, so that the gaps opened by the planets in the disk can overlap. We find that these conditions are not satisfied by any known extra-solar system of multiple planets. This is consistent with these planets having suffered a significant migration, that brought them close to the parent star where they could be discovered. We predict that systems similar to the Jupiter-Saturn case in terms of mass ratio and separation will be discovered only when it will be possible to detect distant planets that did not migrate substantially.

The results of this paper support the view, proposed in Tsiganis et al. (2005) and Gomes et al. (2005), that the giant planets of the solar system, at the end of the gas disk era, were on orbits with small mutual separation. However, from the quantitative point of view, supporting the initial conditions adopted in the model of Tsiganis et al. and Gomes et 
al. is problematic. We suggest that a late fast growth of Saturn's mass or, more likely, a late enhancement of the viscosity towards the end of the disk's lifetime, could have extracted Saturn from the 2:3 resonance with Jupiter and driven it close to the 1:2 resonance. We supported this scenario with simulations, but which are nevertheless qualitative, given our limited knowledge of process of planet growth and of disk disappearance.

We are grateful to Frederic Masset for his suggestions and for a careful reading of this manuscript. We also thank the two anonymous reviewers for their constructive suggestions. We are grateful to the National Program of Planetology for support.

\section{REFERENCES}

Balbus, S. A., Hawley, J. F. 1991. A powerful local shear instability in weakly magnetized disks. I - Linear analysis. II - Nonlinear evolution. Astrophysical Journal $376,214-233$.

Bate, M. R., Lubow, S. H., Ogilvie, G. I., Miller, K. A. 2003. Three-dimensional calculations of high- and low-mass planets embedded in protoplanetary discs. Monthly Notices of the Royal Astronomical Society 341, 213-229.

Crida, A., Morbidelli, A., Masset, F. 2006. On the width and shape of gaps in protoplanetary disks. Icarus 181, 587-604.

Crida, A., Morbidelli, A., Masset, F. 2007. Simulating planet migration in globally evolving disks. Astronomy and Astrophysics 461, 1173-1183.

Crida, A., Morbidelli, A., 2007. Cavity opening by a giant planet in a protoplanetary disk and effects on planetary migration. MNRAS, under revision. 
Gammie, C. F. 1996. Layered Accretion in T Tauri Disks. Astrophysical Journal 457, 355.

Goldreich, P., Tremaine, S. 1980. Disk-satellite interactions. Astrophysical Journal $241,425-441$.

Gomes, R., Levison, H. F., Tsiganis, K., Morbidelli, A. 2005. Origin of the cataclysmic Late Heavy Bombardment period of the terrestrial planets. Nature 435, 466-469.

Guillot, T., Hueso, R. 2006. The composition of Jupiter: sign of a (relatively) late formation in a chemically evolved protosolar disc. Monthly Notices of the Royal Astronomical Society 367, L47-L51.

Haghighipour, N., Boss, A. P. 2003. On Gas Drag-Induced Rapid Migration of Solids in a Nonuniform Solar Nebula. Astrophysical Journal 598, 1301-1311.

Hayashi C. 1981. Structure of the solar nebula, growth and decay of magnetic fields and effects of magnetic and turbulent viscosities on the nebula. Prog. Theor. Phys. Suppl., 70, 35-53.

Ilgner, M., Nelson, R. P. 2006a. On the ionisation fraction in protoplanetary disks. I. Comparing different reaction networks. Astronomy and Astrophysics 445, 205-222.

Ilgner, M., Nelson, R. P. 2006b. On the ionisation fraction in protoplanetary disks. II. The effect of turbulent mixing on gas-phase chemistry. Astronomy and Astrophysics $445,223-232$.

Kley, W. 1999. Mass flow and accretion through gaps in accretion discs. Monthly Notices of the Royal Astronomical Society 303, 696-710.

Kley, W., D’Angelo, G., Henning, T. 2001. Three-dimensional Simulations of a Planet Embedded in a Protoplanetary Disk. Astrophysical Journal 547, 457-464. 
Levison H. F., Morbidelli, A., Gomes, R. and Tsiganis, K. 2007 Origin of the structure of the Kuiper Belt during a Dynamical Instability in the Orbits of Uranus and Neptune. Icarus, submitted.

Lin, D. N. C., Papaloizou, J. 1979. Tidal torques on accretion discs in binary systems with extreme mass ratios. Monthly Notices of the Royal Astronomical Society 186, 799-812.

Lin, D. N. C., Papaloizou, J. 1986. On the tidal interaction between protoplanets and the protoplanetary disk. III - Orbital migration of protoplanets. Astrophysical Journal 309, 846-857.

Lubow, S. H., Seibert, M., Artymowicz, P. 1999. Disk Accretion onto High-Mass Planets. Astrophysical Journal 526, 1001-1012.

Lynden-Bell, D., Pringle, J. E. 1974. The evolution of viscous discs and the origin of the nebular variables.. Monthly Notices of the Royal Astronomical Society 168, 603-637.

Malhotra, R. 1993. The Origin of Pluto's Peculiar Orbit. Nature 365, 819.

Masset, F. 2000a. FARGO: A fast eulerian transport algorithm for differentially rotating disks. Astronomy and Astrophysics Supplement Series 141, 165-173.

Masset, F. S. 2000b. FARGO: A Fast Eulerian Transport Algorithm for Differentially Rotating Disks. ASP Conf. Ser. 219: Disks, Planetesimals, and Planets 219, 75.

Masset, F., Snellgrove, M. 2001. Reversing type II migration: resonance trapping of a lighter giant protoplanet. Monthly Notices of the Royal Astronomical Society 320, L55-L59. 
Masset, F. S., Papaloizou, J. C. B. 2003. Runaway Migration and the Formation of Hot Jupiters. Astrophysical Journal 588, 494-508.

Masset, F. S., Morbidelli, A., Crida, A., Ferreira, J. 2006. Disk Surface Density Transitions as Protoplanet Traps. Astrophysical Journal 642, 478-487.

Masset, F. S. 2006. Planet-disk interactions. In Tidal Interactions in Composite Systems M.-J. Goupil and J.-P. Zahn (eds) EAS Publications Series,in press.

Morbidelli, A., Levison, H. F., Tsiganis, K., Gomes, R. 2005. Chaotic capture of Jupiter's Trojan asteroids in the early Solar System. Nature 435, 462-465.

Papaloizou, J., Lin, D. N. C. 1984. On the tidal interaction between protoplanets and the primordial solar nebula. I - Linear calculation of the role of angular momentum exchange. Astrophysical Journal 285, 818-834.

Pollack, J. B., Hubickyj, O., Bodenheimer, P., Lissauer, J. J., Podolak, M., Greenzweig, Y. 1996. Formation of the Giant Planets by Concurrent Accretion of Solids and Gas. Icarus 124, 62-85.

Rice, W. K. M., Wood, K., Armitage, P. J., Whitney, B. A., Bjorkman, J. E. 2003. Constraints on a planetary origin for the gap in the protoplanetary disc of GM Aurigae. Monthly Notices of the Royal Astronomical Society 342, 79-85.

Shakura, N. I., Sunyaev, R. A. 1973. Black holes in binary systems. Observational appearance.. Astronomy and Astrophysics 24, 337-355.

Thommes, E. W. 2005. A Safety Net for Fast Migrators: Interactions between Gap-opening and Sub-Gap-opening Bodies in a Protoplanetary Disk. Astrophysical Journal 626, 1033-1044. 
Tsiganis, K., Gomes, R., Morbidelli, A., Levison, H. F. 2005. Origin of the orbital architecture of the giant planets of the Solar System. Nature 435, 459-461.

Varnière, P., Blackman, E. G., Frank, A., Quillen, A. C. 2006. Planets Rapidly Create Holes in Young Circumstellar Disks. Astrophysical Journal 640, 1110-1114.

Ward, W. R. 1986. Density waves in the solar nebula - Differential Lindblad torque. Icarus $67,164-180$.

Ward, W. R. 1997. Protoplanet Migration by Nebula Tides. Icarus 126, 261-281. 


\section{Figure captions}

Fig. 1 An illustration of the dynamical evolution described in MS01. The black and grey curves show the evolutions of the semi major axes of Jupiter and Saturn respectively. Capture in the 2:3 MMR occurs when the migration of Saturn is reversed.

Fig. 2 The same as Fig. 1, but for an initial location of Saturn close (but not into) the 2:3 MMR with Jupiter.

Fig. 3 The evolution of Jupiter (lower set of curves starting at $r=1$ ) and Saturn (upper set of curves, starting at $r=1.4)$. Different grey levels refer to different aspect ratios, as labeled. The viscosity is independent of radius and equal to $10^{-5.5}$ in all cases. The planets are released after 400 orbits.

Fig. 4 The dash-dotted curve shows the initial density profile of the disk, adopted in all simulations. The solid curves show the density profile corresponding to the moment when the planets are released, at 1 and 1.4 respectively for Jupiter and Saturn. Different grey levels refer to different aspect ratios, as labeled. The viscosity is independent of radius and equal to $10^{-5.5}$ in all cases.

Fig. 5 The evolution of Jupiter and Saturn in two simulations, both with $H / r=3 \%$ and $\nu=10^{-5.5}$. The black curves refer to the simulation adopting the initial disk surface density shown in Fig. 5. The grey curves adopt an initial surface density profile that has been divided by a factor of 2 . The planets are released in the two cases after about 400 orbital periods at $r=1$. The gray curves are plotted after rescaling the time as $t^{\prime}=(t-400) / 2$. The fact that gray and balck curves overlap shows that the evolution is the same, but the migration speed is reduced proportionally with the mass of the disk. 
Fig. 6 The surface density of the disk in the simulation with $H / r=3 \%$. Different grey levels refer to different times, labeled in unit of initial Jupiter's orbital period. The planets are released at time $T=400$.

Fig. 7 The evolution of Jupiter and Saturn in three simulations, with $H / r=3 \%$ and $\nu=10^{-5.5}$. The simulation plotted in black and labeled 'Hill' is the one already shown in Fig. 3, The simulation reported in grey and labeled ' $\mathrm{H}$ ' adopts a different prescription for the smoothing length, which is now imposed equal to $70 \%$ of the local thickness of the disk. The simulation plotted in light grey and labeled 'High res.' is the same as the latter simulation, but with azimuthal and radial grid resolutions increased by a factor of 2 . Time is measured relative to the instant $T_{0}$ when Saturn starts to migrate outward. The semi major axes of the planets are normalized relative to the semi major axis of Jupiter at $T_{0}$. This allows a more direct comparison among the three simulations.

Fig. 8 The evolution of Jupiter and Saturn in two simulations, with $H / r=4 \%$, $\nu=10^{-5.5}$. The simulation represented by black curves, labelled ' 0.6 Hill', is the one already shown in Fig. 3, obtained adopting a smoothing length $\rho=0.6 R_{H}$. That represented by grey curves, labelled ' 0.25 Hill' has been obtained with $\rho=0.25 R_{H}$. Finally, the simulation represented by light grey curves, labelled 'Excl. Hill' has also been obtained with $\rho=0.25 R_{H}$, but excluding the torques exerted on the planets by the gas inside their Hill spheres.

Fig. 9 The evolution of Jupiter and Saturn in two simulations, with $H / r=4 \%$, $\nu=10^{-5.5}$ and $\rho=0.7 R_{H}$. In the simulation plotted in black and labeled 'Nominal', the planets have been released on free-to-evolve orbits after 400 orbital periods at $r=1$. In the simulation reported in grey and labeled 
'Late release' the planets have been released after 5,000 orbits. Notice that the grey curves have been shifted downwards by $1 \%$, in order to avoid a perfect overlap with the black curves. Therefore, evolution of the planets after the release time is the same.

Fig. 10 The evolution of Jupiter (lower set of curves starting at $r=1$ ) and Saturn (upper set of curves, starting at $r=1.4)$. Different grey levels refer to different viscosities, as labeled. The aspect ratio $H / r$ is equal to $4 \%$ in all cases. The planets are released after 400 initial Jovian orbits.

Fig. 11 The density profiles of the disk at the moment when the planets are released. Different grey levels refer to different viscosities, as labeled. The aspect ratio is $4 \%$ in all simulations.

Fig. 12 The grey curves show the evolutions of Saturn (starting at 1.4) and Jupiter (starting at 1 ) in the simulation with $H / r=3 \%, \nu=10^{-5.5}$ and $\rho=0.7 H$. Time is counted in initial Jovian orbital periods, from the instant when the planets are released. The black curves show how the evolutions of Saturn and Jupiter change, starting from $t=900$, in the case where Saturn (but not Jupiter) is allowed to accrete mass with a removal rate of 1 .

Fig. 13 The evolution of two planets with 3 Jupiter masses (starting at $r=1$ ) and 1 Jupiter masses (starting at $r=1.4$ ). The disk aspect ratio is $3 \%$ and the viscosity is $10^{-5.5}$, independent of radius. A smoothing length equal to $70 \%$ of the local disk's height is used.

Fig. 14 The black solid curves show the evolution of Jupiter and Saturn. As usual, the planets start at $r=1$ and 1.4 respectively, and are released after 400 initial Jovian orbital periods. The disk scale heigh is $5 \%$ and the viscosity is $10^{-5.5}$. After that the planets 
lock in the 2:3 MMR (at $t \sim 900$ the planets' semi major axes remain substantially constant. The grey curves show the location of the 2:3 and 1:2 MMR with Jupiter, according to Kepler law. Notice that the semi major axis of Saturn is slightly larger than that corresponding to the 2:3 MMR in the Kepler approximation, due to the effects of the disk's gravity. At $t=3200$ (marked by a vertical dashed line), the viscosity of the disk is increased at a rate of $6.28 \times 10^{-9}$ per orbital period. This eventually forces Jupiter to migrate inward and extracts Saturn from the 2:3 MMR. The simulation is stopped when Saturn reaches the vicinity of the 1:2 MMR with Jupiter. 


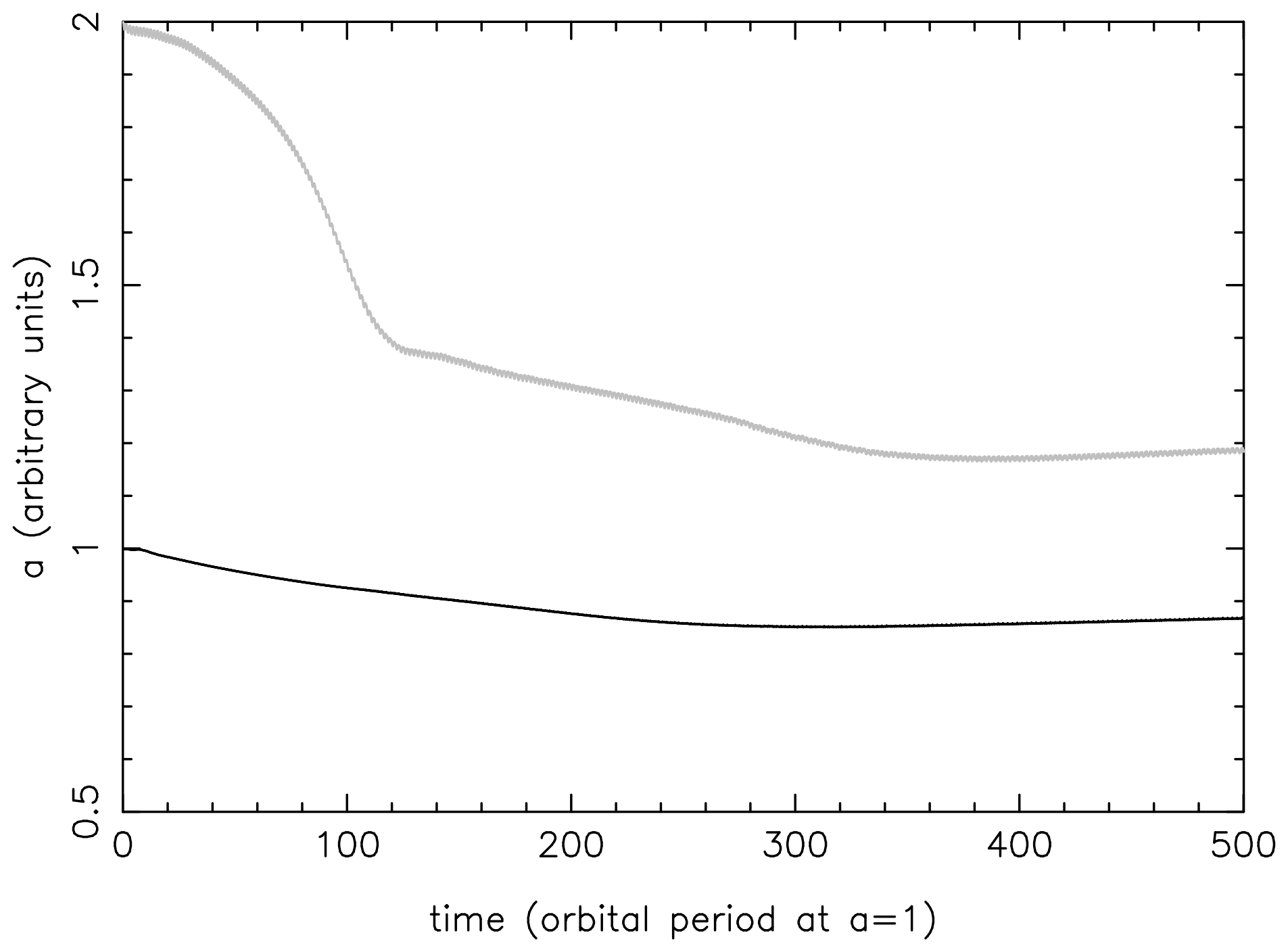

Fig. 1.- 


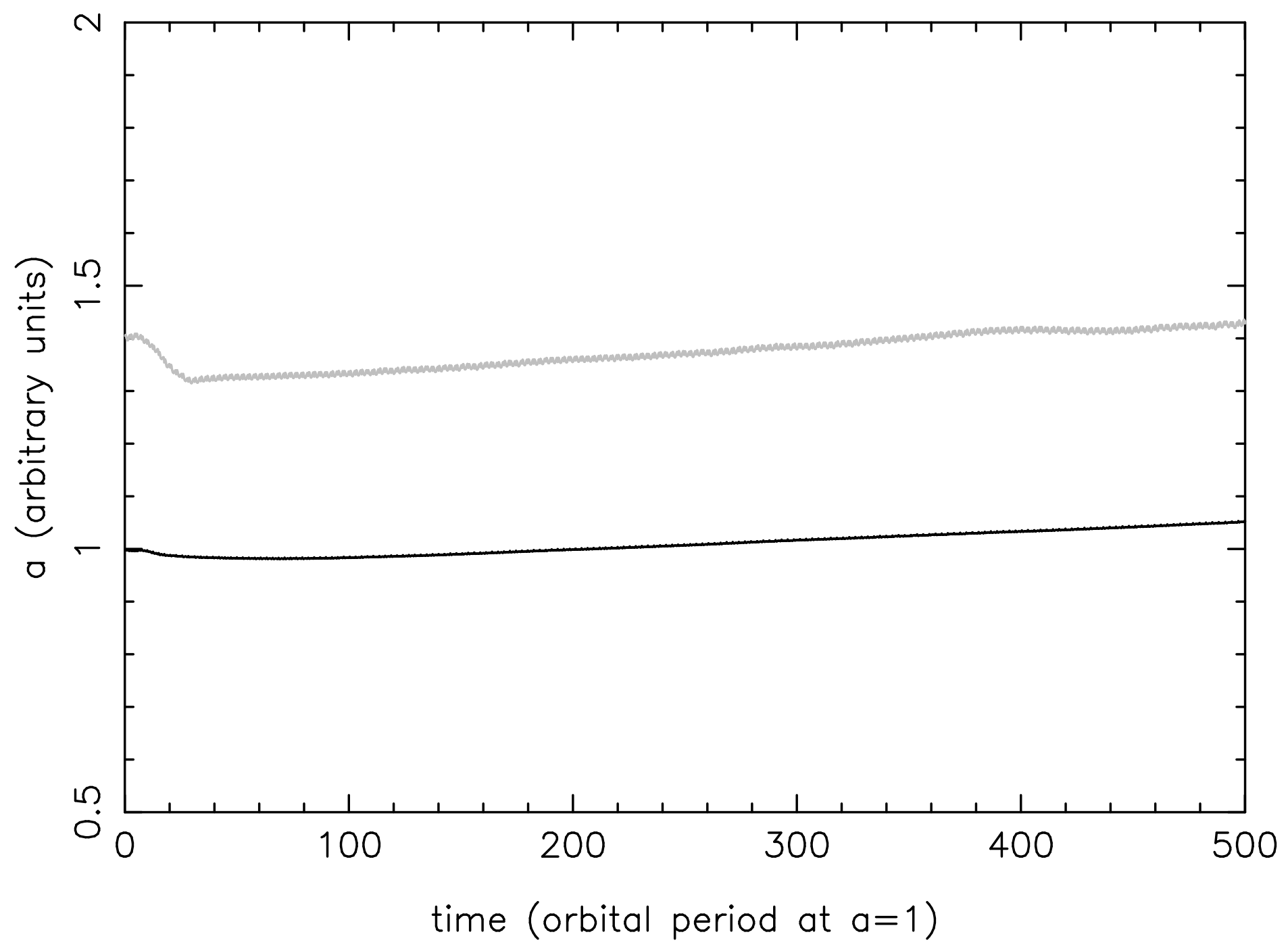

Fig. 2.- 


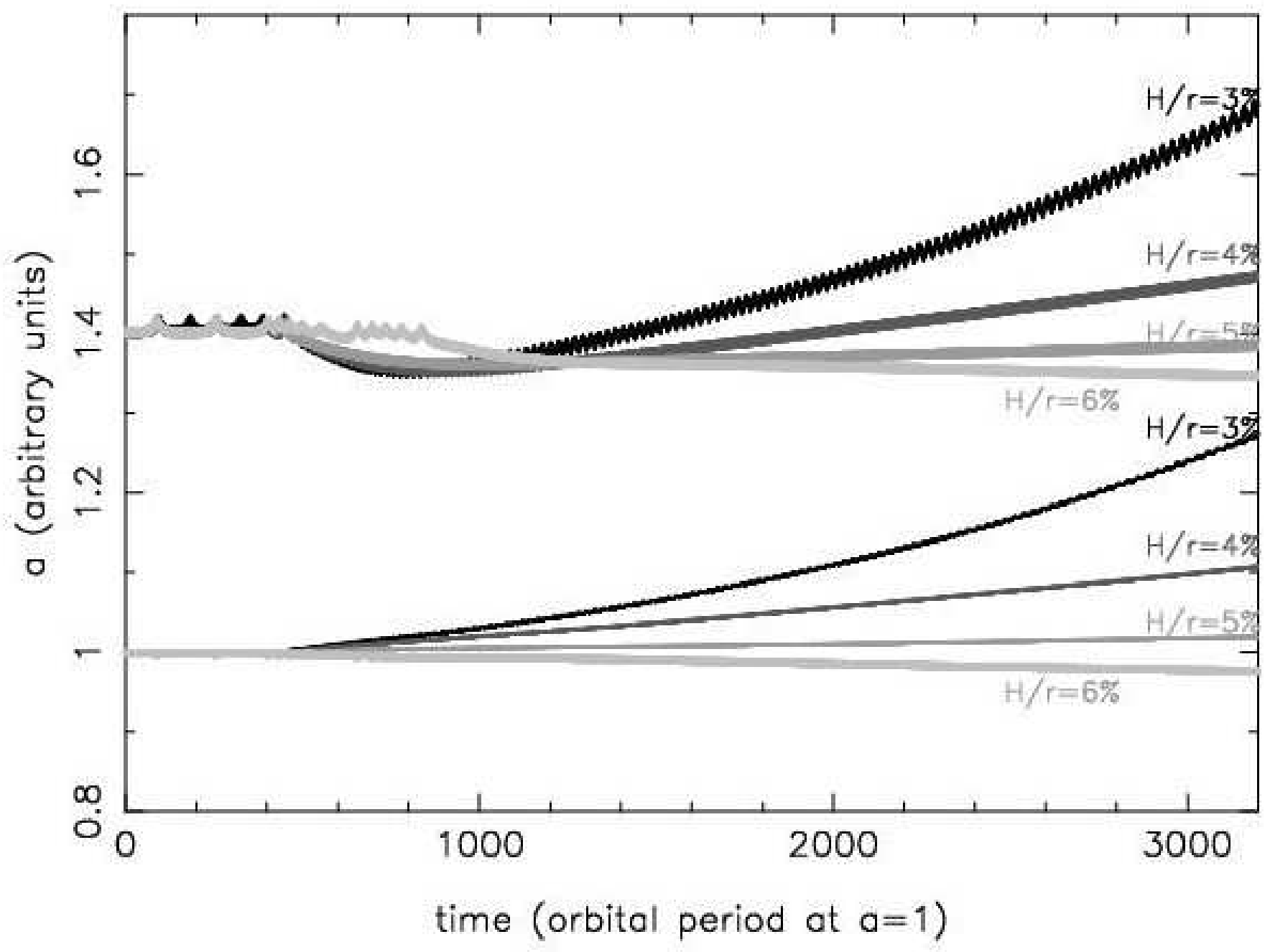

Fig. 3.- 


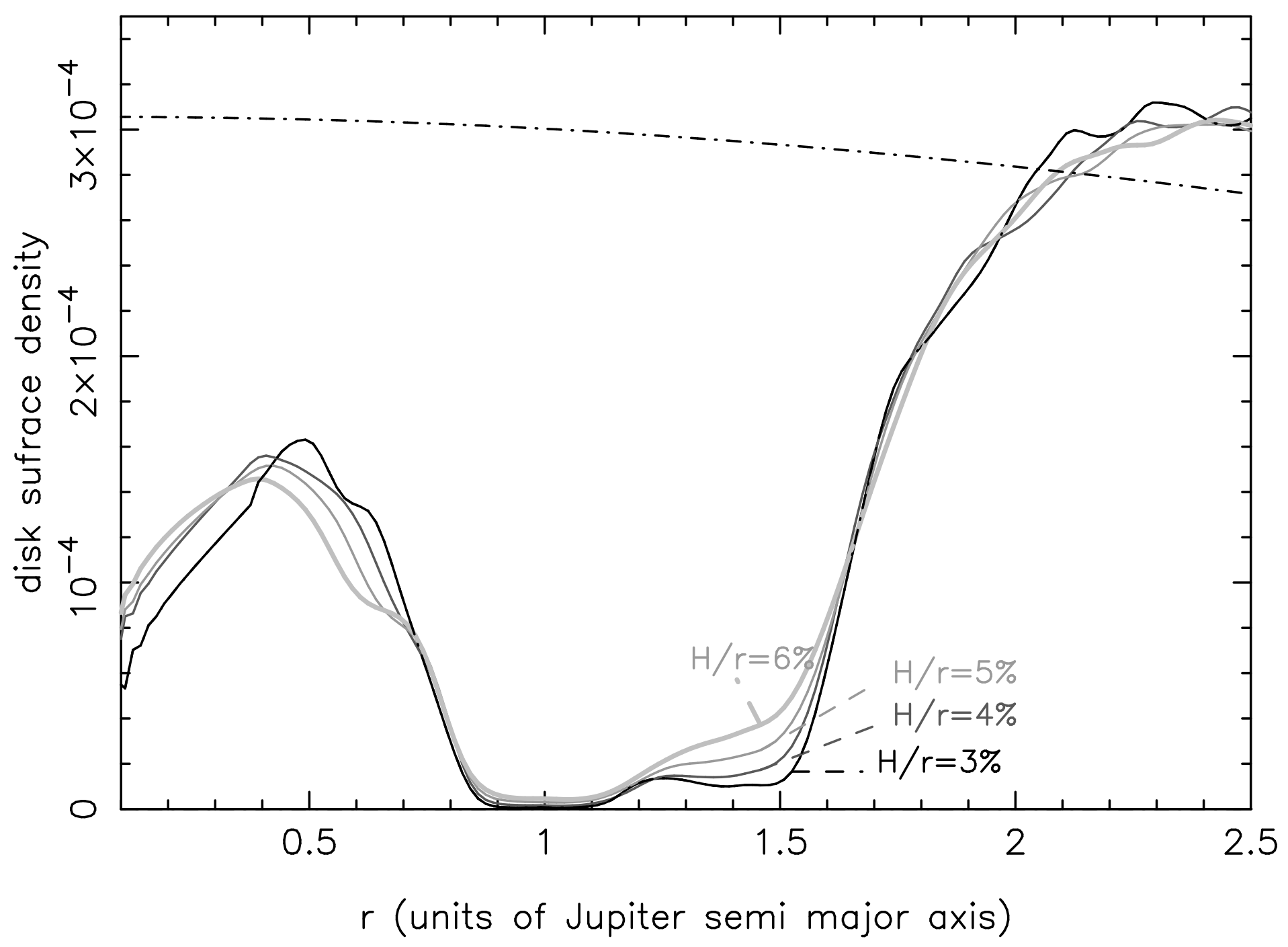

Fig. 4.- 


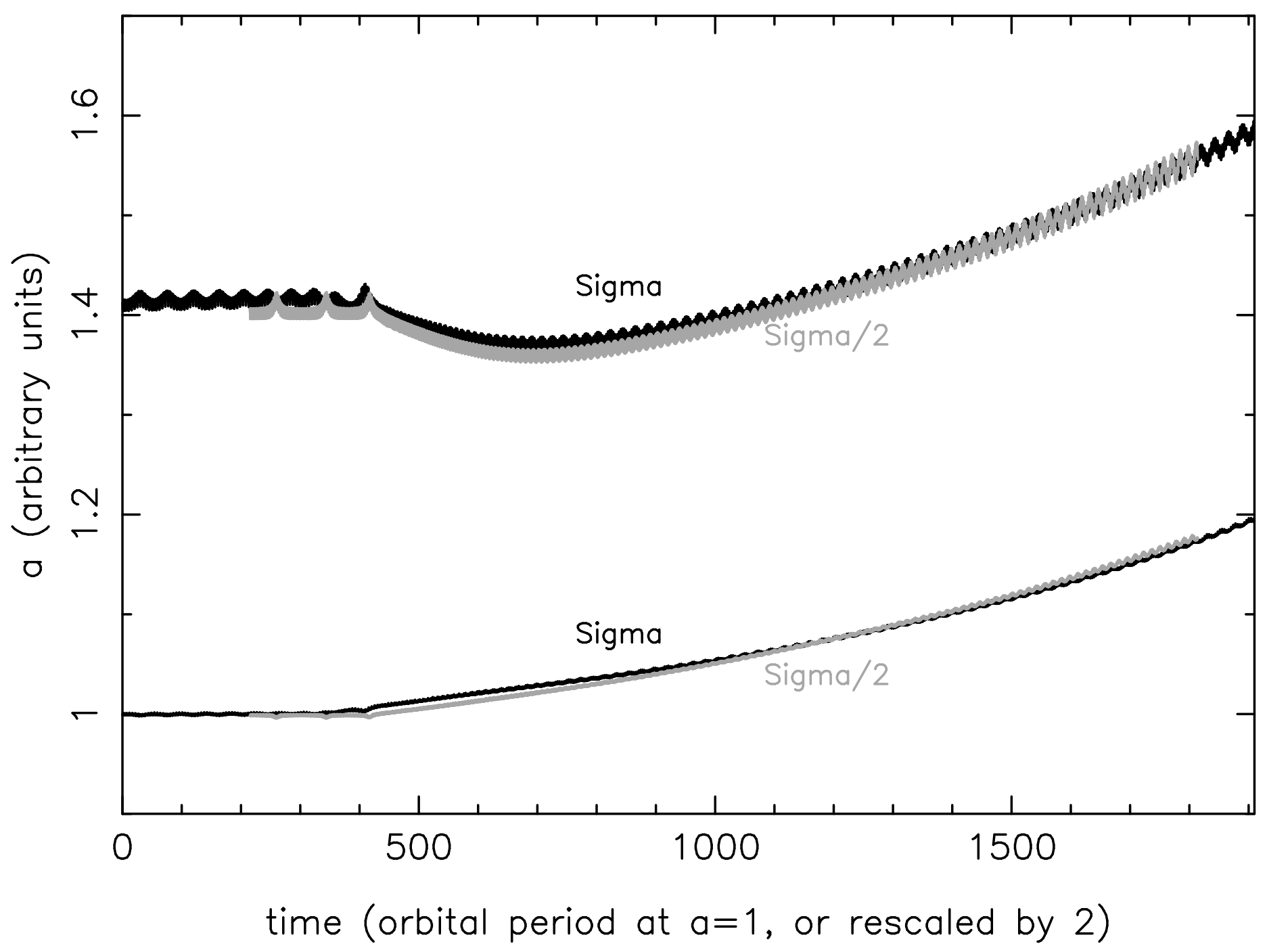

Fig. 5.- 


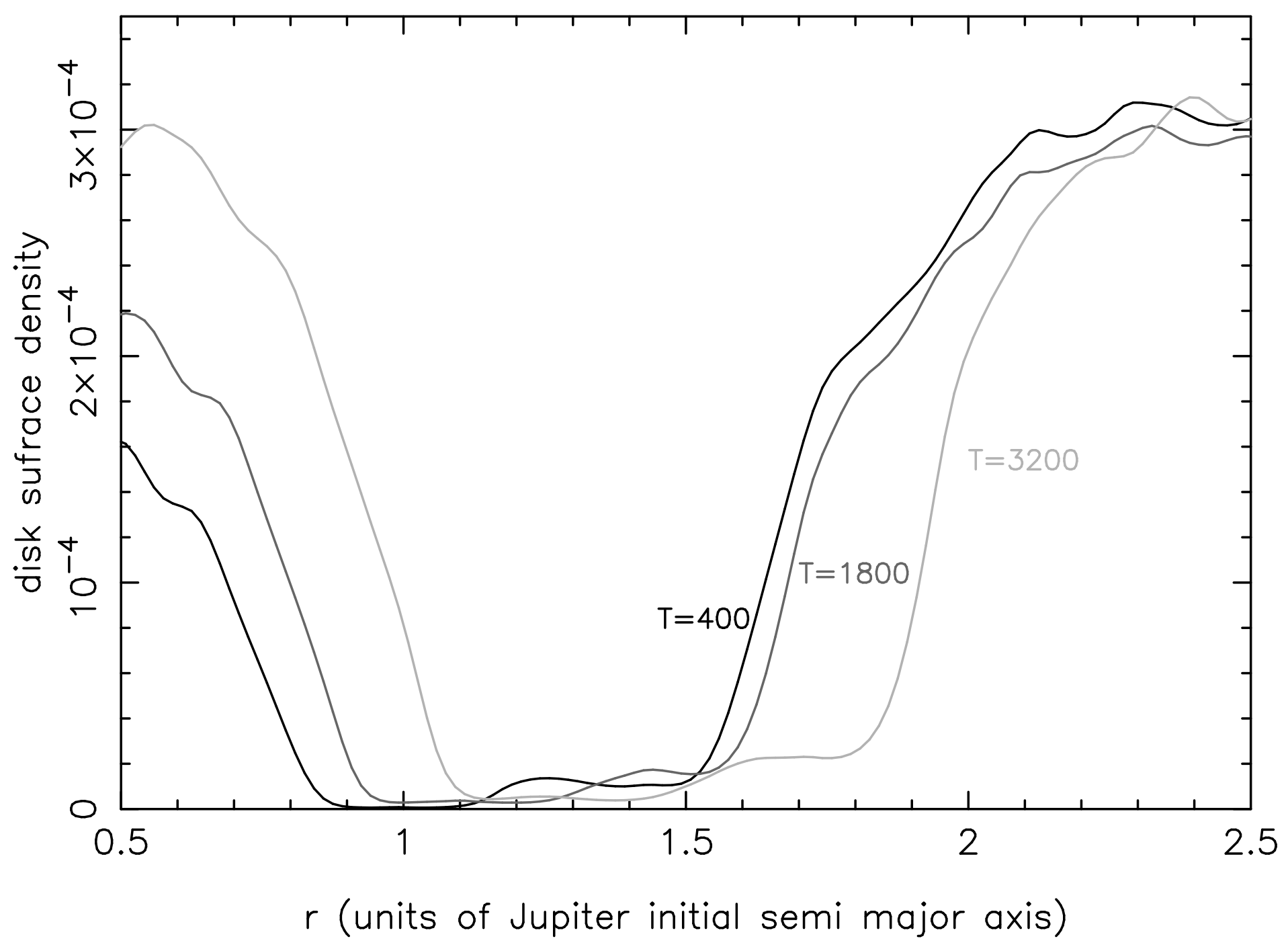

Fig. 6.- 


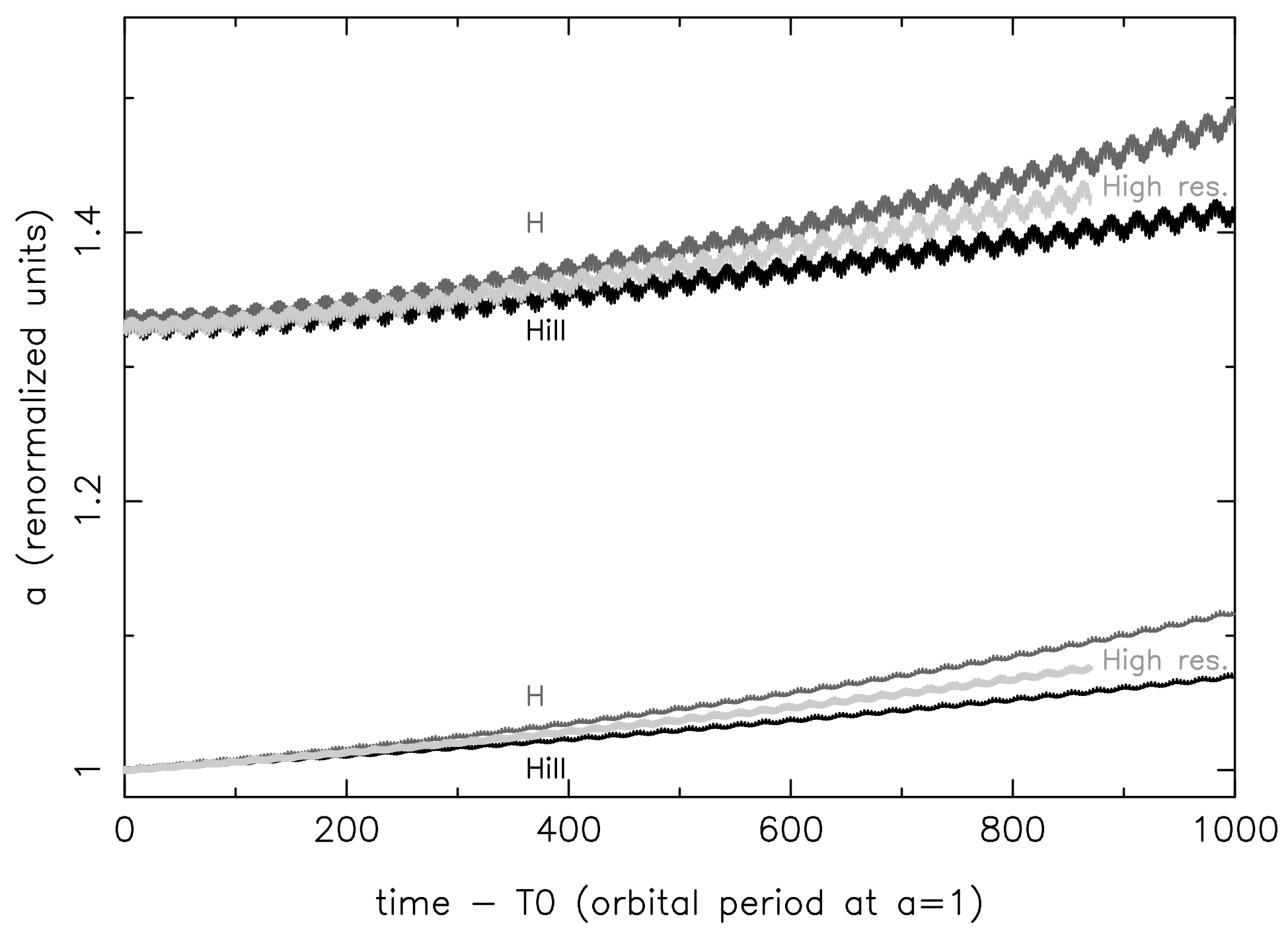

Fig. 7.- 


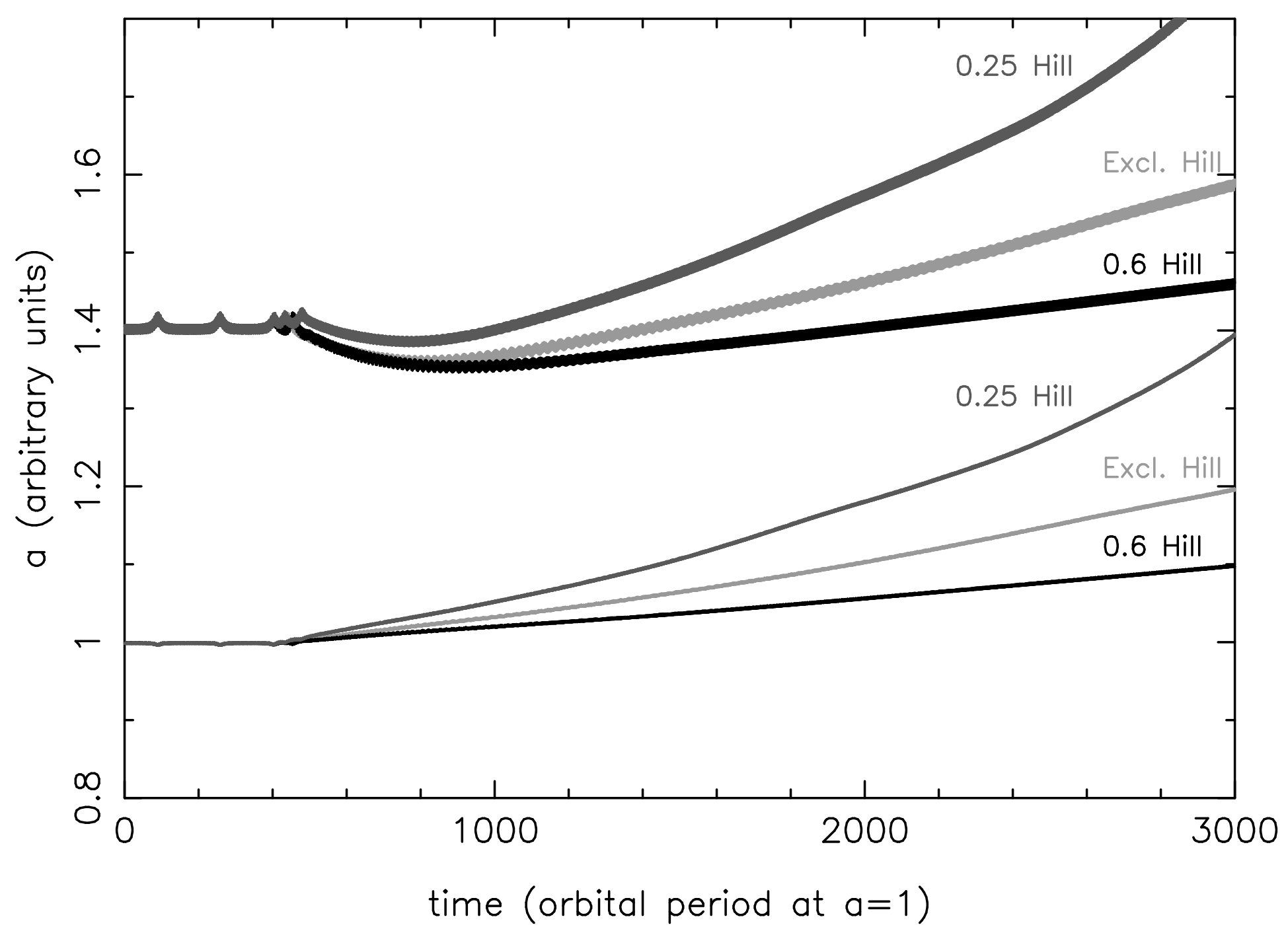

Fig. 8.- 


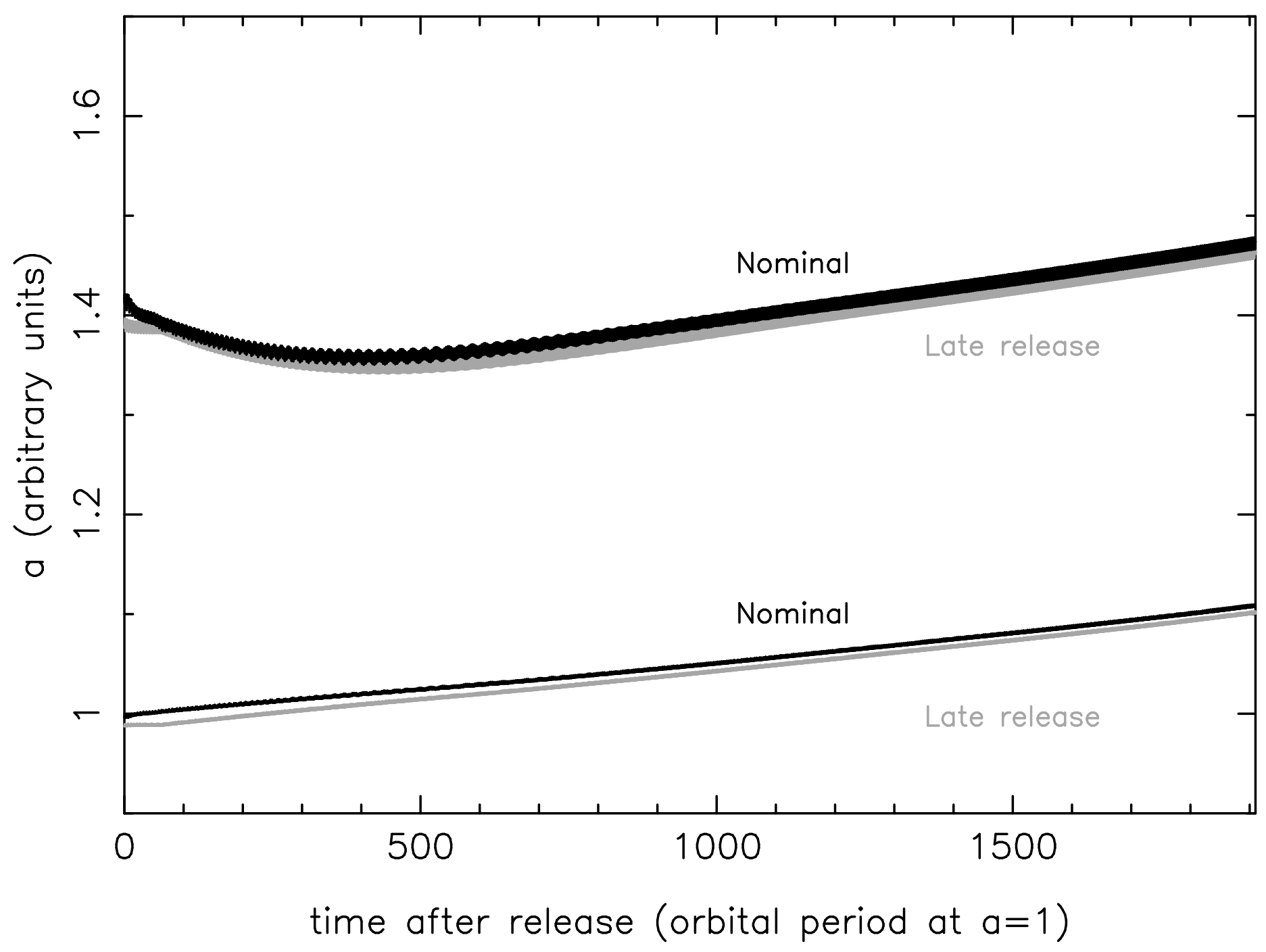

Fig. 9.- 


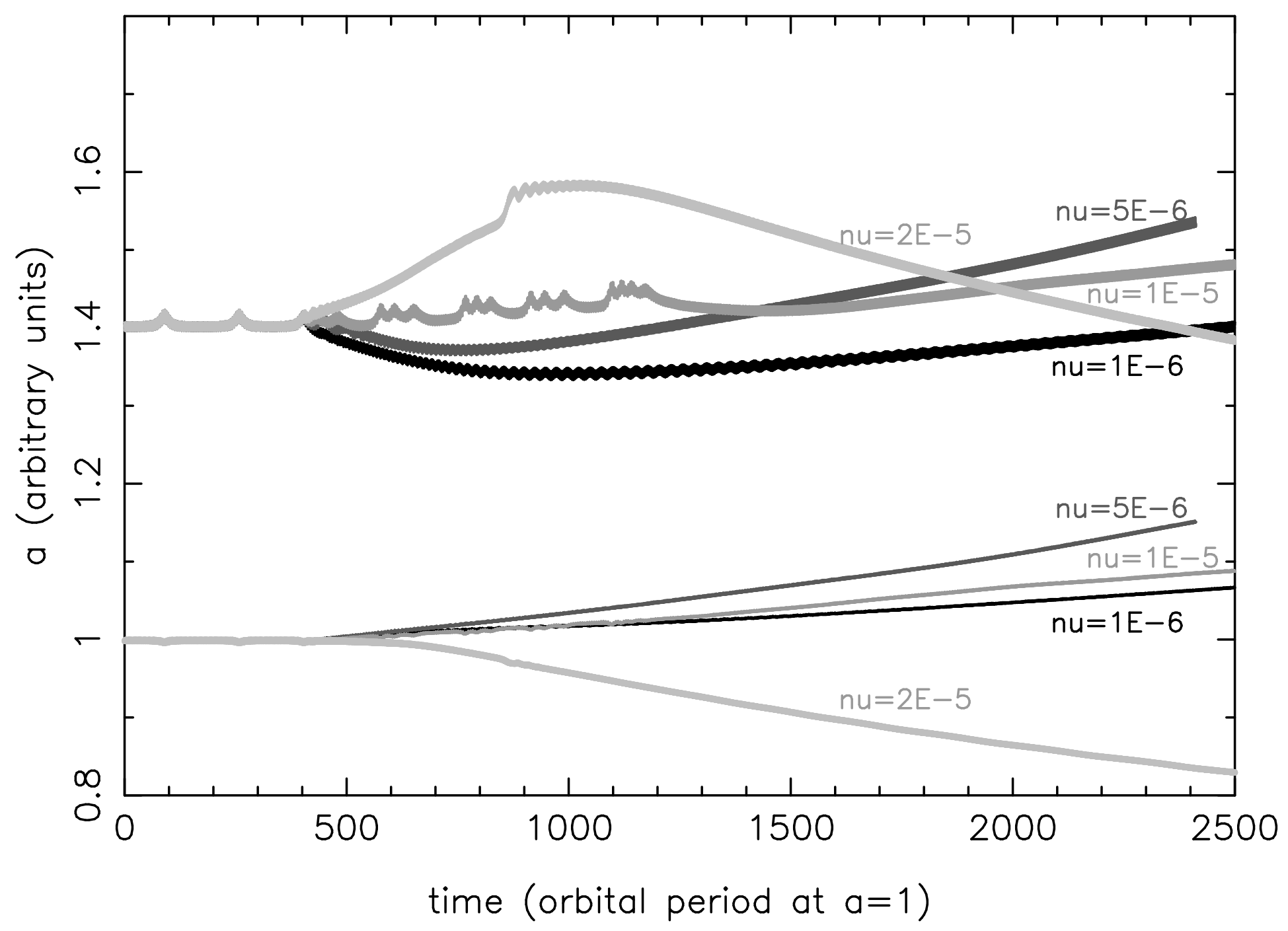

Fig. 10.- 


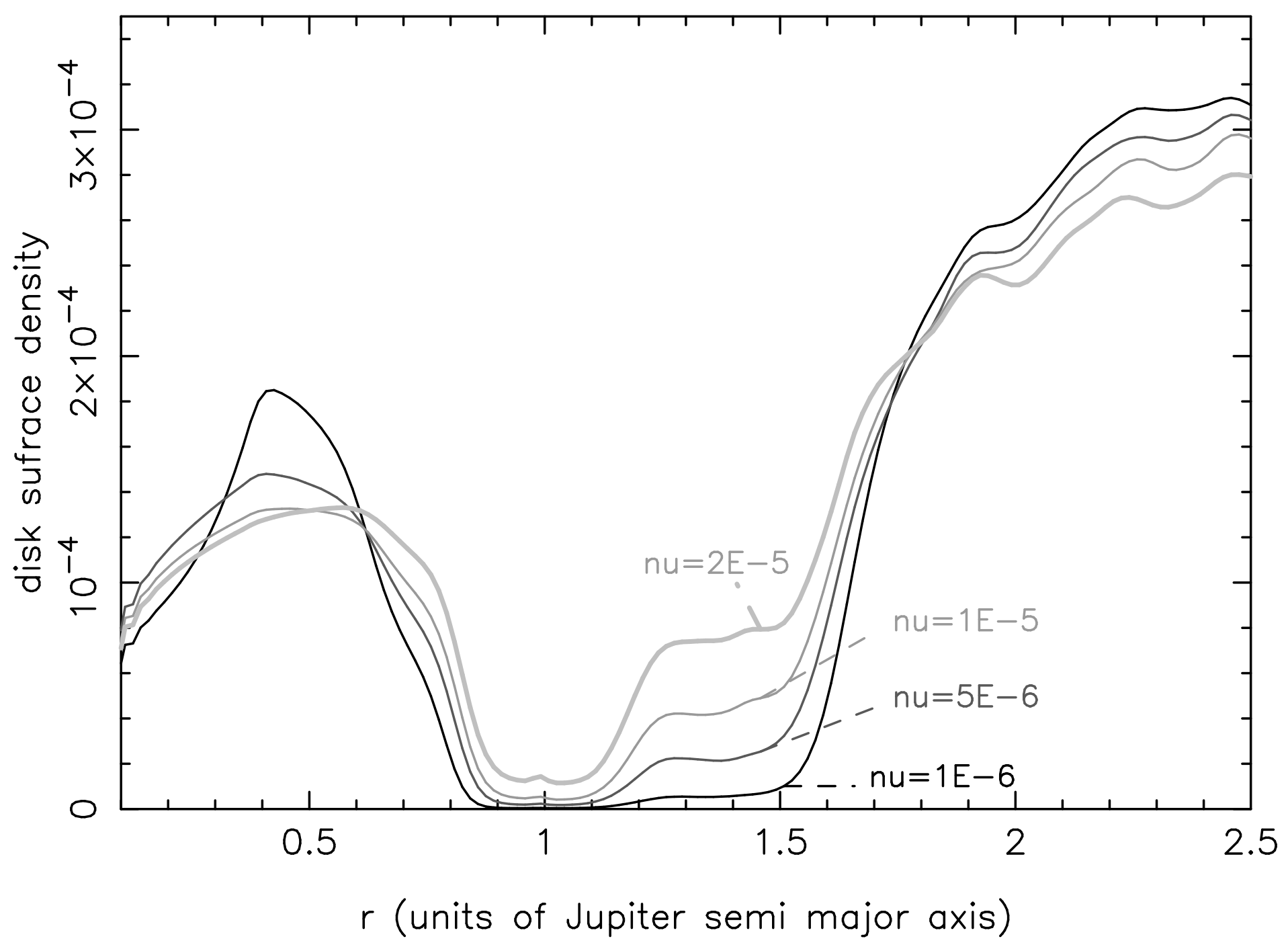

Fig. 11.- 


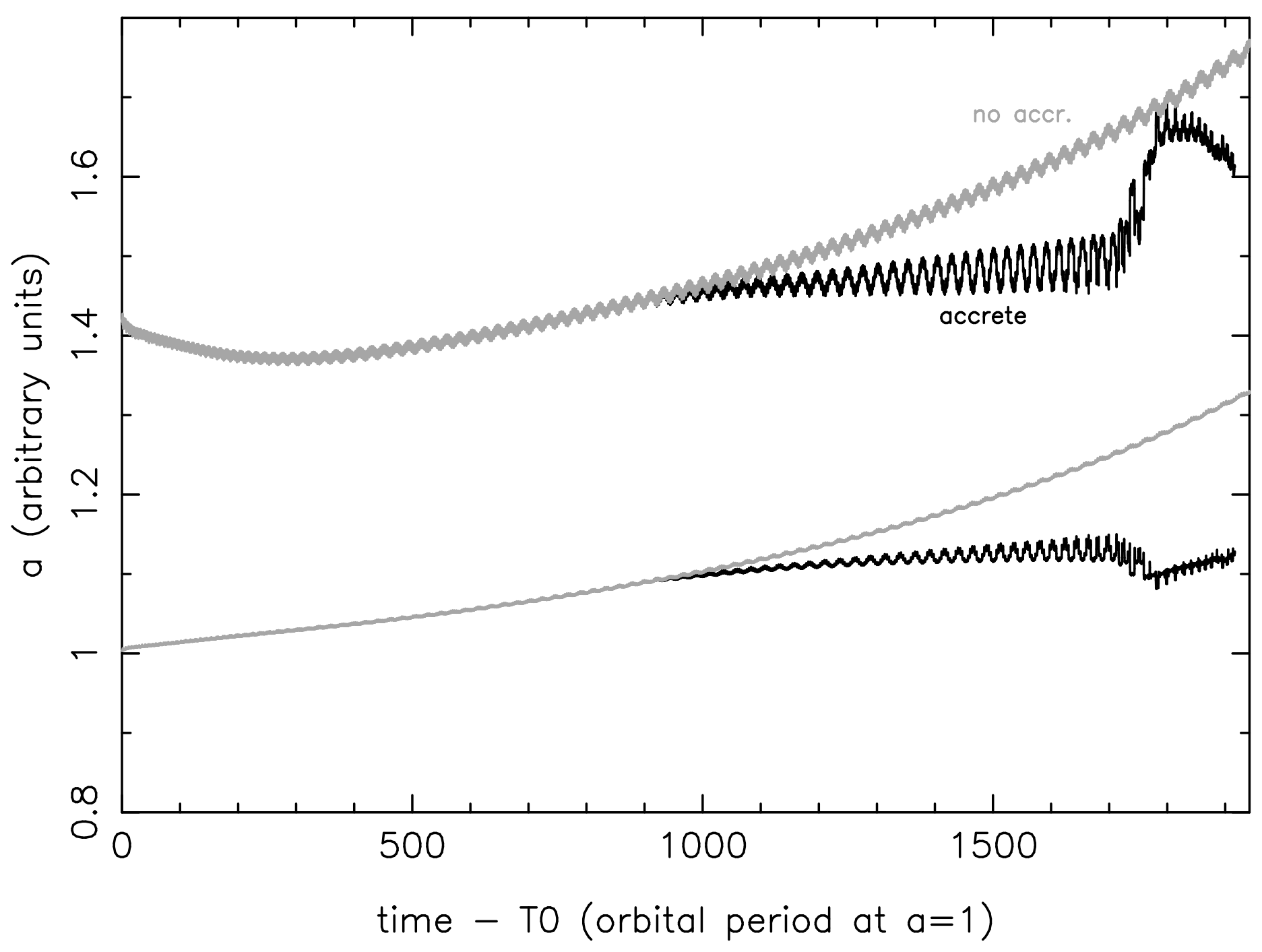

Fig. 12.- 


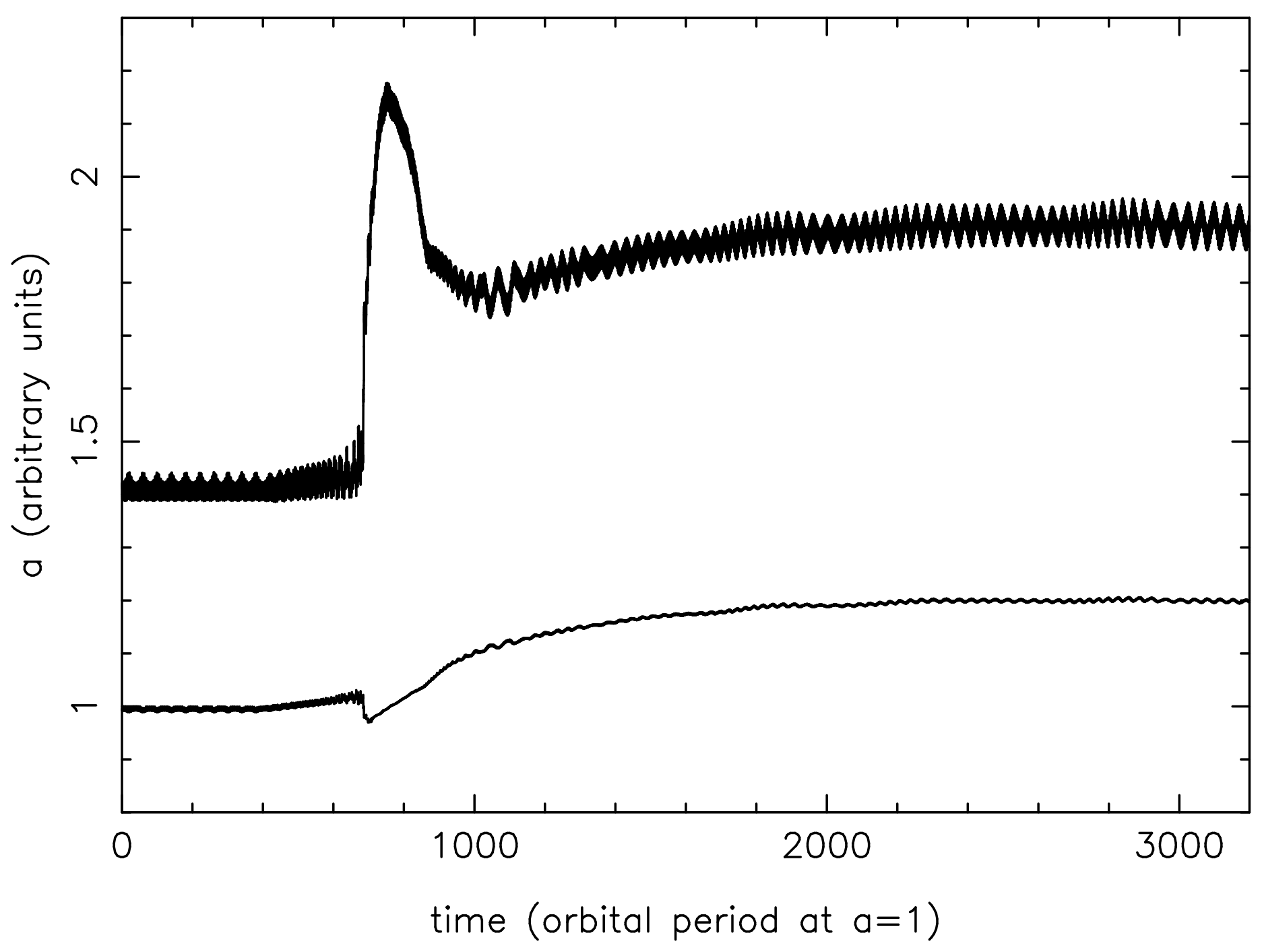

Fig. 13.- 


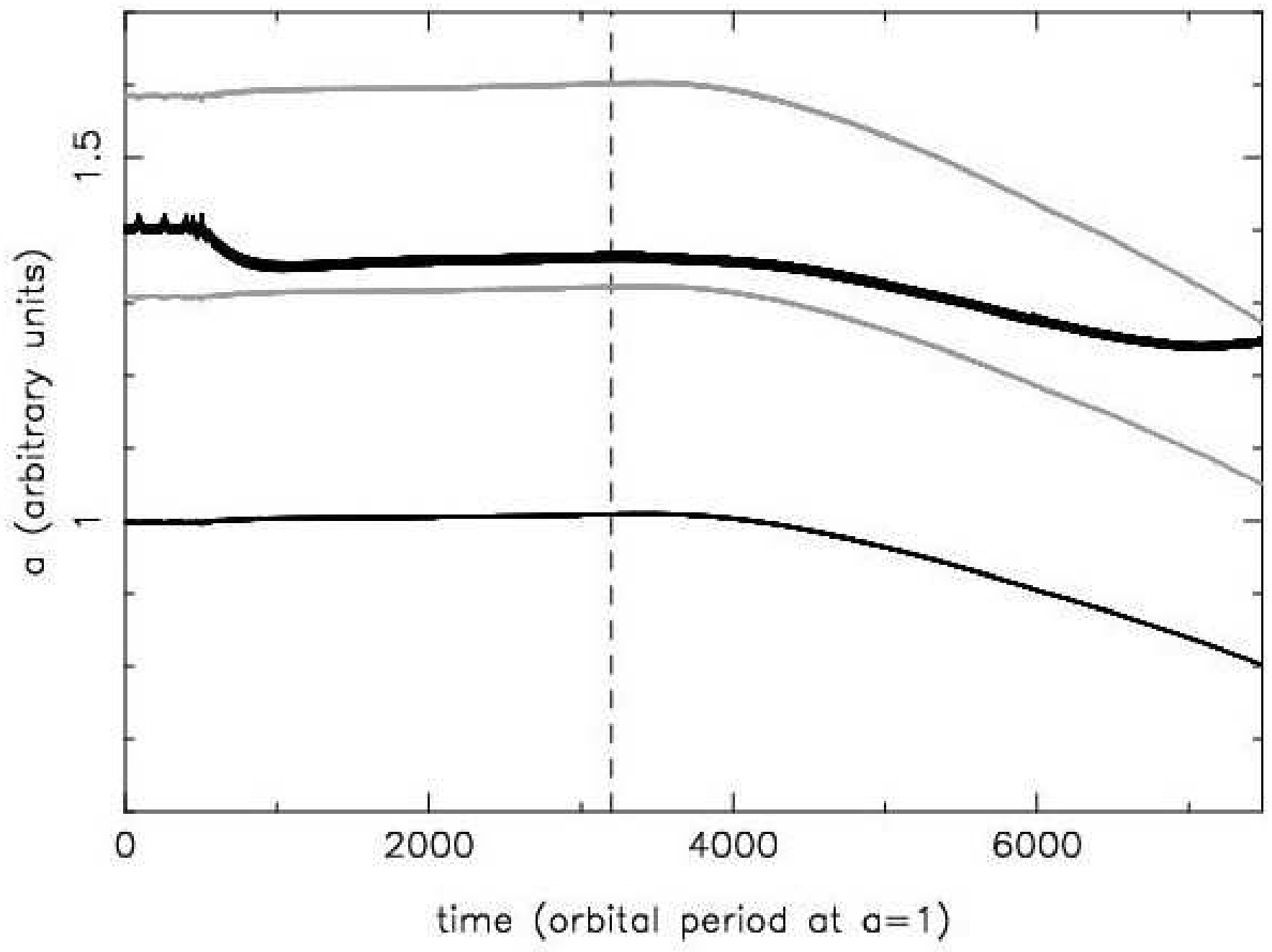

Fig. 14.- 